Draft version November 5, 2018

Preprint typeset using $\mathrm{L}^{A} \mathrm{~T}_{\mathrm{E}} \mathrm{X}$ style emulateapj v. 11/10/09

\title{
THE 2006-2007 ACTIVE PHASE OF ANOMALOUS X-RAY PULSAR 4U 0142+61: RADIATIVE AND TIMING CHANGES, BURSTS, AND BURST SPECTRAL FEATURES
}

\author{
Fotis P. Gavrill ${ }^{1}{ }^{2}$, Rim DiB ${ }^{3}$, Victoria M. Kaspi ${ }^{3}$ \\ Draft version November 5, 2018
}

\begin{abstract}
After at least 6 years of quiescence, Anomalous X-ray Pulsar (AXP) 4U 0142+61 entered an active phase in 2006 March that lasted several months and included six X-ray bursts as well as many changes in the persistent X-ray emission. The bursts, the first seen from this AXP in $>11$ years of Rossi X-ray Timing Explorer monitoring, all occurred in the interval between 2006 April 6 and 2007 February 7. The burst durations ranged from $0.4-1.8 \times 10^{3} \mathrm{~s}$. The first five burst spectra are well modeled by blackbodies, with temperatures $k T \sim 2-9 \mathrm{keV}$. However, the sixth burst had a complicated spectrum that is well characterized by a blackbody plus two emission features whose amplitude varied throughout the burst. The most prominent feature was at $14.0 \mathrm{keV}$. Upon entry into the active phase the pulsar showed a significant change in pulse morphology and a likely timing glitch. The glitch had a total frequency jump of $(1.9 \pm 0.4) \times 10^{-7} \mathrm{~Hz}$, which recovered with a decay time of $17 \pm 2$ days by more than the initial jump, implying a net spin-down of the pulsar. Within the framework of the magnetar model, the net spin-down of the star could be explained by regions of the superfluid that rotate slower than the rest. The bursts, flux enhancements, and pulse morphology changes can be explained as arising from crustal deformations due to stresses imposed by the highly twisted internal magnetic field. However, unlike other AXP outbursts, we cannot account for a major twist being implanted in the magnetosphere.
\end{abstract}

Subject headings: — stars: neutron - X-rays: stars - X-rays: bursts — pulsars: individual $(4 \mathrm{U} 0142+61)$

\section{INTRODUCTION}

It is now generally accepted that the class of objects referred to as "Anomalous X-ray Pulsars" (AXPs) are magnetars - young isolated neutron stars powered by the evolution of their high magnetic fields (Thompson \& Duncan 1995; Thompson \& Duncan 1996). AXPs are X-ray pulsars with periods in the range $2-12 \mathrm{~s}$, and X-ray luminosities $\left(\sim 10^{33}-\right.$ $10^{35} \mathrm{erg} \mathrm{s}^{-1}$ ) that cannot be accounted for by their available spin-down energy. The magnetar model was first proposed to explain the dramatic behavior exhibited by an apparently different object class - the Soft Gamma Repeaters (SGRs). SGRs show persistent properties similar to AXPs, but they were first discovered by their enormous bursts of soft gamma rays $\left(>10^{44} \mathrm{erg}\right)$ and their much more frequent, shorter, and thus less energetic bursts of hard X-rays. To date, SGR-like X-ray bursts have been observed from six AXPs, thus solidifying the connection between the two source classes (Gavriil et al.|2002; Kaspi et al.|2003; Woods et al. 2005; Krimm et al. 2006; Mereghetti et a. 2009; Kumar \& Safi-Harb 2011). For reviews of magnetar candidates and AXPs see Woods \& Thompson (2006), Kaspi (2007), and Mereghetti (2008).

Thus far, only the magnetar model can explain the bursts observed from SGRs and AXPs

\footnotetext{
${ }^{1}$ NASA Goddard Space Flight Center, Astrophysics Science Division, Code 662, Greenbelt, Maryland, 20771, USA

${ }^{2}$ Center for Research and Exploration in Space Science and Technology, University of Maryland Baltimore County, 1000 Hilltop Circle, Baltimore, Maryland, 21250, USA

${ }^{3}$ Department of Physics, McGill University, Montreal, QC, H3A 2T8, Canada.
}

(Thompson \& Duncan 1995). The internal magnetic field exerts stresses on the crust which can lead to large scale rearrangements of the external field, which we observe as giant flares. If the stress is more localized, then it can fracture the crust and displace the footpoints of the external magnetic field which results in short X-ray bursts. The highly twisted internal magnetic field also slowly twists up the external field; magnetospheres of magnetars may therefore be globally twisted (Thompson et al. 2002). Reconnection in this twisted magnetosphere has also been proposed as an additional mechanism for the short bursts (Lyutikov 2002).

In addition to bursts, AXPs and SGRs exhibit pulsed and persistent flux variations on multiple different timescales. An hours-long increase in the pulsed flux has been seen to follow a burst in AXP 1E 1048.1-5937 (Gavriil et al. 2006). On longer timescales, AXPs can exhibit abrupt increases in flux which decay on severalweek timescales. These occur in conjunction with bursts and have been suggested as being due to thermal radiation from the stellar surface after the deposition of heat from bursts. Such flux enhancements have been observed in SGRs (see Woods et al. 2001, for example). The flux enhancement of AXP 1E 2259+586 during its 2002 outburst can also interpreted as burst afterglow (Woods et al. 2004), however, a magnetospheric interpretation is also plausible (Zhu et al. 2008). AXP 1E 1048.1-5937 exhibited three unusual flux 'flares.' In the first two, the pulsed flux rose on week-long timescales and subsequently decayed back on time scales of months (Gavriil \& Kaspi 2004; Tam et al. 2008). These variations have been tentatively attributed to twists implanted in the external magnetosphere from stresses 
on the crust imposed by the internal magnetic field. AXPs XTE J1810-197, 1E 1547.0-5408, and the AXP candidate AX J1845-0258 have also exhibited large flux variations (Ibrahim et al. 2004; Halpern et al. 2008; Gotthelf \& Vasisht 1998; Tam et al. 2006), however it is not clear whether these were of the abrupt rise type as in $1 \mathrm{E} 2259+586$ or the slow-rise type as in $1 \mathrm{E} 1048.1$ 5937. CXO J164710.2-455216 showed a clear abrupt rise (Muno et al. 2007). 1RXS J1708-4009 has been argued to have flux variations associated with timing events (Israel et al. 2007b). Finally, AXP 4U 0142+61 has exhibited the longest timescale flux variations thus far, in which the pulsed flux increased by $29 \pm 8 \%$ over a period of 2.6 years (Dib et al. 2007; Gonzalez et al. 2010).

$4 \mathrm{U} 0142+61$ is an 8.7 -s AXP. It has a period derivative of $\dot{P}=0.2 \times 10^{-11}$, implying a surface dipole magnetic field of $1.3 \times 10^{14} \mathrm{G}$. $4 \mathrm{U} 0142+61$ was monitored by RXTE in 1997 and from 2000 to 2007. Gavriil \& Kaspi (2002) showed that $4 \mathrm{U} 0142+61$ generally rotates with high stability. Morii. Kawai, \& Shibazaki (2005) reported a possible timing glitch in 1999 on the basis of an Advanced Satellite for Cosmology and Astrophysics $(A S C A)$ observation in which the value of the frequency was marginally discrepant with the frequency as reported by Gavriil \& Kaspi (2002). Dib et al. (2007) showed that the glitch may have occurred but is not required by the existing data. Dib et al. (2007) also reported on the evolution of the properties of $4 \mathrm{U} 0142+61$ from 2000 March to 2006 April. In particular they reported stable timing, and an evolution of the pulse profile in $2-4 \mathrm{keV}$ where the dip between the two peak was rising between 2000 and 2006. They also reported a $29 \pm 8 \%$ increase in the pulsed flux between 2002 May and 2004 December. As of 2006 March, in the published flux history of this source, there had been no reports of any X-ray activity such as bursts or flares, as described above for other AXPs.

Here we report on the first detection of bursts from AXP 4U 0142+61, making this the seventh AXP for which this phenomenon has been observed. We also report that the source appears to have entered an active phase in 2006 March in which almost every aspect of the emission changed. Our observations are described in Section 2 Our burst, pulsed morphology, pulse phase, pulsed flux, and timing analysis are presented, respectively, in Sections 3.1, 3.2, 3.3, 3.4, and 3.5. In Section 4, we discuss the possible origins of this behavior and the implications for the magnetar model.

\section{OBSERVATIONS}

$4 \mathrm{U} 0142+61$ has been monitored with Rossi X-ray Timing Explorer (RXTE) in 1997 and from 2000 to 2007. It has been monitored bi-monthly since 2005 March, with a typical observation length of $\sim 5$ ks. On 2006 March 23 , the source entered an active phase where many pulsed flux, spectral, and pulse profile changes were observed. We detected 6 bursts in three observations after the entry into the active phase. After each burst detection, several $R X T E$ target-of-opportunity (ToO) observations were made in addition to the regular monitoring.

Here, we present a detailed analysis of the three observations containing bursts (see Table 1). We also present an analysis of 91 other observations spanning the 2005 March 21 to 2007 May 15 time period (MJD 53481 to MJD 54235, Observation IDs 91070-05-04-00 to 92006-

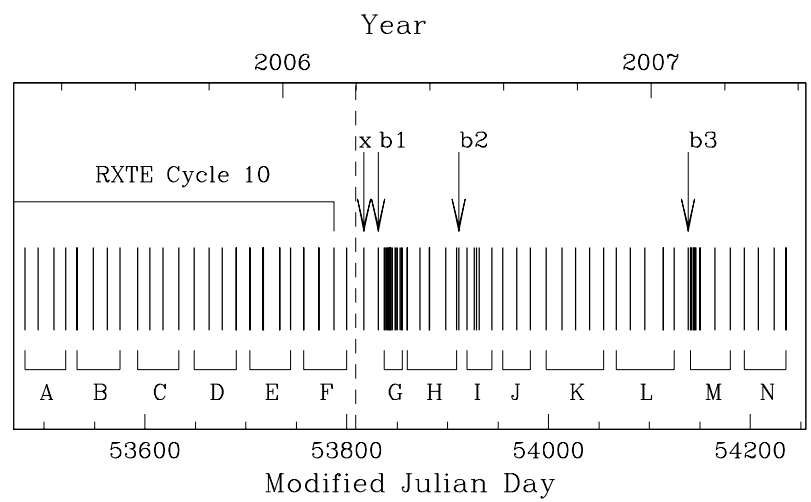

FIG. 1.- Epochs of the RXTE observations of $4 \mathrm{U} 0142+61$ analyzed in this paper (see $\S 3.2$. Each observation is represented by a vertical line. The dashed line marks the entry of the source into the active phase. An " $x$ " marks the first observation where the pulse profile was significantly different from the long-term average. "b1", "b2", and "b3" mark the observations containing bursts. To the left of the dotted line, the letters refer to groups of observations having similar total integration times. To the right, the letters refer to groups of observations having similar pulse profiles.

05-32-00). 32 of these observations were prior to the entry into the active phase, and the remaining 59 observations were after. This long-term analysis was performed in order to track the evolving pulsed flux and timing properties of the source.

Figure 1 shows a timeline of the 94 analyzed observations. Note that two segments of any observation that was split counted as separate observations if the segments were given different observation IDs. The ranges of epochs with an increased density of observations contain the ToO observations. Prior to the active phase, the groups of observations referred to by capital letters in the Figure have similar total integration times. The groups of observations in the active phase have similar pulse profiles. These groups will be referred to in Sections 3.2 and 3.5 .

\section{ANALYSIS AND RESULTS}

All data presented here are from the Proportional Counter Array (PCA; Jahoda et al. 2006) aboard RXTE. The PCA is made up of five identical and independent proportional counter units (PCUs). Each $\mathrm{PCU}$ is a Xenon/methane proportional counter with a propane veto layer. The data were collected in either GoodXenonwithPropane or GoodXenon mode which record photon arrival times with $\sim 1-\mu$ s resolution and bins them with 256 spectral channels in the $\sim 2-60 \mathrm{keV}$ band. For all RXTE observations of $4 \mathrm{U} 0142+61$, photon arrival times at each epoch were adjusted to the solar system barycenter using the position obtained by Patel et al. (2003) from Chandra X-ray Observatory data.

\subsection{Burst Analysis}

For each monitoring observation of $4 \mathrm{U} 0142+61$, using software that can handle the raw telemetry data, we generated 31.25-ms lightcurves using all Xenon layers and events in the $2-20 \mathrm{keV}$ band. These lightcurves were searched for bursts using our automated burst search algorithm introduced in Gavriil et al. (2002) and discussed further in Gavriil et al. (2004). In an observation on 2006 
TABLE 1

4U 0142+61 Burst Observation Log and Burst Properties

\begin{tabular}{|c|c|c|c|c|c|c|}
\hline \multicolumn{7}{|c|}{$\overline{\text { Observations Containing Bursts }}$} \\
\hline Observation ID & $92006-05-03-00$ & & $92006-05$ & $\overline{09-01}$ & & $92006-05-25-00$ \\
\hline Burst Date & 6 April 2006 & & 26 June & 2006 & & 7 February 2007 \\
\hline Burst MJD (UTC) & 53831 & & 5391 & & & 54138 \\
\hline Number of Active PCUs & $3^{\mathrm{a}}$ & & 3 & & & 2 \\
\hline Number of Bursts & 1 & & 4 & & & 1 \\
\hline \multicolumn{7}{|c|}{ Burst Temporal and Spectral Properties $^{b}$} \\
\hline Burst Number & 1 & 2 & 3 & 4 & 5 & 6 \\
\hline$t_{p}{ }^{\mathrm{c}}(\mathrm{UT})$ & $07: 09.55 .544(7)$ & $01: 15: 54.555(7)$ & $01: 15: 55.119(58)$ & $01: 16: 9.216(7)$ & $01: 20: 0.131(3)$ & 10:04:43.264(60) \\
\hline$\tau_{r} \mathrm{~d}, \mathrm{i}(\mathrm{ms})$ & $7_{-2}^{+3}$ & $7_{-2}^{+2}$ & $58_{-16}^{+19}$ & $7_{-4}^{+3}$ & $3_{-2}^{+1}$ & $60_{-2}^{+9}$ \\
\hline$\tau_{f} \mathrm{e}, \mathrm{i}(\mathrm{ms})$ & $33_{-20}^{+13}$ & $18_{-13}^{+10}$ & $65_{-40}^{+32}$ & $188_{-24}^{+118}$ & $228_{-26}^{+207}$ & $3621_{-75}^{+264}$ \\
\hline$\tau_{t} \mathrm{f}, \mathrm{i}_{(\mathrm{s})}$ & $4.2(2)$ & $0.22(4)$ & $12.21(6)$ & $191.6(4)$ & $40.7(2)$ & $824.9(8)$ \\
\hline$f_{p}{ }^{\mathrm{g}, \mathrm{i}}($ counts $/ \mathrm{s} / \mathrm{PCU})$ & $543_{-258}^{+174}$ & $852_{-358}^{+273}$ & $151_{-39}^{+28}$ & $329_{-40}^{+194}$ & $246_{-27}^{+214}$ & $551_{-11}^{+39}$ \\
\hline$f_{p} \mathrm{~g}, \mathrm{i}, \mathrm{k}\left(10^{-10} \mathrm{erg} / \mathrm{s} / \mathrm{cm}^{2}\right)$ & $187_{-89}^{+60}$ & $144_{-61}^{+46}$ & $61_{-16}^{+11}$ & $31_{-4}^{+18}$ & $52_{-6}^{+45}$ & $66_{-1}^{+\frac{1}{+}}$ \\
\hline$f_{t}{ }^{\mathrm{h}, \mathrm{i}}($ counts/s/PCU) & $13.3(8)$ & $125(21)$ & $25.5(1)$ & $8.47(1)$ & $7.14(3)$ & $12.70(1)$ \\
\hline$f_{t} \mathrm{~h}, \mathrm{i}, \mathrm{k}\left(10^{-10} \mathrm{erg} / \mathrm{s} / \mathrm{cm}^{2}\right)$ & $4.6(3)$ & $21(4)$ & $10.38(4)$ & $0.7992(9)$ & $1.516(6)$ & $1.518(1)$ \\
\hline$F_{\text {tot }}{ }^{\mathrm{j}}($ counts $/ \mathrm{PCU})$ & $78(4)$ & $47(7)$ & $328(2)$ & $1686(3)$ & $346(2)$ & $12460(13)$ \\
\hline$F_{\text {tot }} \mathrm{j,k}\left(10^{-10} \mathrm{erg} / \mathrm{cm}^{2}\right)$ & $27(1)$ & $8(1)$ & $134(1)$ & $159.1(2)$ & $73.4(5)$ & $1490(2)$ \\
\hline$T_{90}{ }^{l}(\mathrm{~s})$ & $8.4(4)$ & $0.4(1)$ & $27.5(1)$ & $434(1)$ & $86.7(6)$ & $1757(2)$ \\
\hline Phase $^{\mathrm{m}}$ (cycles) & $0.503(7)$ & $0.487(4)$ & $0.54(2)$ & $0.173(4)$ & $0.749(4)$ & $0.358(4)$ \\
\hline$k T^{\mathrm{n}}(\mathrm{keV})$ & $8.8_{-3.8}^{+12.3}$ & $5.4_{-1.2}^{+1.7^{\prime}}$ & $4.8_{-0.3}^{+0.3}$ & $2.4_{-0.2}^{+0.2}$ & $2.7_{-0.2}^{+0.2}$ & $2.5_{-0.2}^{+0.2}$ \\
\hline$R^{\circ}(\mathrm{m})$ & $24_{-19}^{+20}$ & $163_{-50}^{+61}$ & $100_{-8}^{+.8}$ & $107_{-15}^{+17}$ & $132_{-15}^{+16}$ & $151_{-20}^{+22}$ \\
\hline$\chi^{2} / \mathrm{DoF}{ }^{\mathrm{p}}[\mathrm{DoF}]$ & $0.78[7]$ & $0.87[3]$ & $1.09[38]$ & $0.49[37]$ & $0.81[32]$ & $1.00[48]$ \\
\hline
\end{tabular}

a One PCU switched on and another one off part way through the observation, but the total number of active PCUs stayed constant throughout the observation.

b All quoted errors represent $1-\sigma$ uncertainties. In the fits, the column density was held fixed at $N_{H}=0.64 \times 10^{22} \mathrm{~cm}^{-2}$ (Durant \& van Kerkwijk 2006b)

c Time of burst peak.

d Rise Time.

e Short-term decay time of the tail of the burst.

f Long-term decay time of the tail of the burst.

g 2-60 kev peak flux.

h $2-60 \mathrm{keV}$ flux in the tail component of the burst.

i These parameters were obtained by fitting the model given by Eq. 1 to the burst.

j 2-60 keV fluence. These values were obtained by using Eq. 2

$\mathrm{k}$ All quoted fluxes and fluences in CGS units are unabsorbed.

${ }^{1} T_{90}$ duration, defined as the time it took to collect $90 \%$ of the total burst fluence. These values were obtained by using Eq. 3

$\mathrm{m}$ The phases are relative to the template shown in Figure 7

n Blackbody temperature. For burst 6 , the temperature is that of the blackbody component after accounting for the apparent spectral features (see 3.1 .2 .

o The blackbody radius was calculated assuming a distance to the source of $3.5 \mathrm{kpc}$ (Durant \& van Kerkwijk $2006 \mathrm{a})$.

p Reduced $\chi^{2}$ for the spectral fits.

April 6, we detected a significant burst, and four more bursts were detected in a single observation on 2006 June 25. The sixth and most energetic burst was detected on 2007 February 7. The bursts were significant in each active PCU. See Table 1 for the number of active PCUs in each burst observation, as well as for the burst observation epochs.

To analyze these bursts we created event lists in FITS4 format using the standard FTOOLS 5 . For consistency with previous analyses of SGR/AXP bursts we extracted events in the 2-60 keV band. The burst lightcurves are displayed in Figure 2 (LEFT). Our techniques for characterizing the temporal and spectral properties of bursts were discussed in detail in Gavriil et al. (2004) but we repeat them here as some required modification in order address the distinct properties of these bursts.

Before measuring any burst parameters we determined the instrumental background using the FTOOL pcabackest. We extracted a background model lightcurve using the appropriate energy band and num-

\footnotetext{
4 http://fits.gsfc.nasa.gov

5 http://heasarc.gsfc.nasa.gov/docs/software/ftools/
}

ber of PCUs. pcabackest determines the background count rate only on 16-s time intervals, so we interpolated these values by fitting a polynomial of order 6 to the entire observation, which yielded a good fit for each data set.

\subsubsection{Burst Temporal and Energetic Properties}

The burst peak time $\left(t_{p}\right)$ was determined using the method described in Gavriil et al. (2004); Gavriil et al. (2006). Usually, to measure the fluence for SGR and AXP bursts, we subtract the instrumental background for the lightcurve, integrate the light curve and fit the result to a step function with a linear term whose slope is the "local" background rate. The burst fluence in this case is the height of the step function. Although this technique worked well for the first burst, which was a short, isolated event, it was not appropriate for bursts 2, 3 , and 4 because they had overlapping tails, and bursts 4, 5 and 6 had tails that extended beyond the end of the observation. Thus, we opted to determine the best-fit burst profile , $f(t)$, consisting of exponential rises and 
decays,

$$
f(t)= \begin{cases}f_{p} e^{\left(\frac{t-t_{p}}{\tau_{r}}\right)}+b(t)+B & t \leq t_{p} \\ \left(f_{p}-f_{t}\right) e^{-\left(\frac{t-t_{p}}{\tau_{f}}\right)}+f_{t} e^{-\left(\frac{t-t_{p}}{\tau_{t}}\right)} & t>t_{p}, \\ +b(t)+B & \end{cases}
$$

via maximum likelihood testing. The burst peak time $\left(t_{p}\right)$, and the background model $(b(t))$ determined using pcabackest and the "local" background $(B)$ were held fixed at the values determined by the methods outlined above. We fit for the rise time $\left(\tau_{r}\right)$, the peak flux $\left(f_{p}\right)$, the flux of the long-term tail component $\left(f_{t}\right)$, and for the short $\left(\tau_{f}\right)$ and long-term $\left(\tau_{t}\right)$ decay times of the tail of the burst. Figure 2 (LEFT) shows our observed and model burst lightcurves. The binning was chosen such that the flux in the peak bin was equal to the flux determined from our maximum likelihood fits. We then integrated our events to obtain fluence lightcurves. Figure 2 (RIGHT) shows our observed and model burst fluence time series. For both figures, the contribution from neighboring bursts has been subtracted.

As is done for $\gamma$-ray bursts and SGR and AXP bursts, we characterized the burst duration by $T_{90}$, the time when $90 \%$ of the total burst fluence has been collected. From Eq. 1 it follows that the total fluence, $F_{\text {tot }}$, is given by

$$
F_{\text {tot }}=f_{p} \tau_{r}+\left(f_{p}-f_{t}\right) \tau_{f}+f_{T} \tau_{t},
$$

and the $T_{90}$ duration is given by

$$
T_{90}=\tau_{t} \log \left(10 f_{t} \tau_{t} / F_{\text {tot }}\right) .
$$

Although Eq. 2 is exact, Eq. 3 is an approximation that holds very well because $T_{90} \gg \tau_{r}, \tau_{f}$ for all bursts. This fact is made obvious by Figure 2 (RIGHT). All burst temporal parameters are presented in Table 1.

\subsubsection{Burst Spectral Properties}

Burst spectra were extracted using all the counts within their $T_{90}$ interval. We then filtered the spectra to include counts only from the Xenon layers and from all active PCUs other than PCU 0. PCU 0 was ignored because of the loss of its propane layer and due to its frequent breakdowns. Background intervals were extracted from long, hand-selected, featureless intervals prior to the bursts. Response matrices were created using the FTOOL pcarsp. The burst spectra were grouped such that there were at least 10 counts per bin after background subtraction. Burst spectra, background spectra, and response matrices were then read into the spectral fitting package XSPEC $\mathrm{v}$ 12.3.1. The spectra were fit to photoelectrically absorbed blackbodies using the column density found by Durant \& van Kerkwijk (2006b). Only bins in the $2-30 \mathrm{keV}$ band were included in the fits. The blackbody model provided an adequate fit for bursts 1 through 5; model parameters are presented in Table 1.

Extracted over its very long $T_{90}$ interval (see Table 1), the spectrum of Burst 6 was well fit by a simple blackbody, however, there were hints of spectral features. On much shorter timescales, the spectrum of burst 6 was not well modeled by any simple continuum model and

\footnotetext{
6 http://xspec.gsfc.nasa.gov
}

the presence of spectral features is clear (see Fig. 3). We looked closely at the first $8.69 \mathrm{~s}$ (one pulse period) of the burst (see Fig. 3), and determined that a simple continuum model with less than two spectral features was not a statistically good representation of the data. A simple photoelectrically absorbed blackbody yielded a reduced $\chi^{2}$ of 2.1 for 39 degrees of freedom (DoF). Adding a single Gaussian emission lines improved the fit but not sufficiently, yielding reduced $\chi^{2} \mathrm{~s}$ of 1.3 for 36 DoF. Only a photoelectrically absorbed blackbody plus two Gaussian emission lines provided an adequate fit, with reduced $\chi^{2}=1.1$ for 34 DoF. With this best-fit model, we find lines at energies $8.6_{-0.2}^{+0.1}, 14.2_{-0.3}^{+0.3}$. Note, that $8.6 \mathrm{keV}$ line was very narrow, we thus kept its width fixed at the detectors energy resolution, this was not necessary for the $14.2 \mathrm{keV}$ line as it was relatively broad. Our results from fitting different models to the first $8.69 \mathrm{~s}$ of the burst spectrum are summarized in Figure 3, and the parameters returned from our best-fit model are listed in Table 2.

We investigated whether different continuum models could describe the feature rich burst spectrum just as well as a blackbody. We tried a simple power-law model, as well as more complicated continuum models such as a Cutoff Power-law (PLCUT; White et al. 1983) and a Negative/Positive Exponential (NPEX; Mihara 1995). The PLCUT and NPEX models are often used to fit the continuum component of the line rich spectra of accreting X-ray pulsars (see Coburn et al. 2002). All three of our alternate continuum models also required two emission features at similar energies as the blackbody, however they did not provide improved fits. The best alternative to a blackbody was the PLCUT model with two Gaussian emission lines at 8.8 and $14.3 \mathrm{keV}$ with reduced $\chi^{2}=1.1$ for 33 DoF. Notice how this model combination had the same reduced $\chi^{2}$ as the blackbody combination, but required one more free parameter.

We then determined whether the features could be described by absorption rather than emission. We tried three different absorption models: cyclotron absorption, Gaussian absorption (multiplicative model), and simply subtracting Gaussians. We tested all three of these absorption models with all the continuum models described above. No permutation provided a better fit than a blackbody with two emission lines, a simple power-law with two Gaussian absorption lines at $10.8 \mathrm{keV}$ and a broad (Gaussian width $\sigma=4.4 \mathrm{keV}$ ) feature at $2.5 \mathrm{keV}$ being the best absorption model combination with reduced $\chi^{2}=1.2$ for 33 DoF.

To study the temporal evolution of these features we extracted 17.38-s (two pulse periods) long spectra in steps of $4.34 \mathrm{~s}$ (half a pulse period) from the peak of the burst, and repeated the spectral fitting procedure outlined above. Fig. 4 displays the spectral evolution of the features. The middle panel is a surface plot of the change in $\chi$ after subtracting the continuum model (blackbody). A vertical slice in the middle panel corresponds to a spectrum of the burst extracted over a two-pulse-period long interval, and these spectra were extracted in time steps of half a spin-period. The features showed clear temporal variability and were most prominent near the onset of the burst (see Fig. 4). From the surface plot in Fig. 4 (middle panel), we see that the $14.2 \mathrm{keV}$ is highly significant at the start of the burst, and remains detectable 
TABLE 2

Burst 6 Spectral Fit

\begin{tabular}{lc}
\hline \hline \multicolumn{1}{c}{ Parameter } & Value $^{\mathrm{a}}$ \\
\hline Column Density $^{\mathrm{b}}, N_{H}\left(10^{22} \mathrm{~cm}^{-2}\right)$ & 0.64 \\
$2-60 \mathrm{keV}$ Flux $^{\mathrm{c}}\left(10^{-10} \mathrm{erg} \mathrm{s}^{-1} \mathrm{~cm}^{-2}\right)$ & $39.6_{-3.6}^{+3.7}$ \\
Reduced $\chi^{2}$ (Degrees of Freedom) & $1.1(34)$ \\
\hline
\end{tabular}

Blackbody Component

\begin{tabular}{|c|c|}
\hline $\begin{array}{l}\text { Temperature, } k T(\mathrm{keV}) \\
\operatorname{Radius}^{\mathrm{c}}(\mathrm{km}) \\
2-60 \mathrm{keV} \mathrm{Flux}^{\mathrm{c}}\left(10^{-10} \mathrm{erg} \mathrm{s}^{-1} \mathrm{~cm}^{-2}\right)\end{array}$ & $\begin{array}{c}5.8_{-0.3}^{+0.3} \\
0.19_{-0.01}^{+0.02} \\
34.8_{-3.5}^{+3.6}\end{array}$ \\
\hline \multicolumn{2}{|c|}{ Gaussian Emission Line 1} \\
\hline $\begin{array}{l}\text { Energy }(\mathrm{keV}) \\
\text { Widthe }^{\mathrm{e}} \sigma(\mathrm{keV}) \\
2-60 \mathrm{keV} \mathrm{Flux}^{\mathrm{c}}\left(10^{-10} \mathrm{erg} \mathrm{s}^{-1} \mathrm{~cm}^{-2}\right)\end{array}$ & $\begin{array}{c}8.6_{-0.2}^{+0.1} \\
\cdots \\
0.6_{-0.2}^{+0.2}\end{array}$ \\
\hline \multicolumn{2}{|c|}{ Gaussian Emission Line 2} \\
\hline $\begin{array}{l}\text { Energy }(\mathrm{keV}) \\
\text { Width, } \sigma(\mathrm{keV}) \\
2-60 \mathrm{keV} \mathrm{Flux}{ }^{\mathrm{c}}\left(10^{-10} \mathrm{erg} \mathrm{s}^{-1} \mathrm{~cm}^{-2}\right)\end{array}$ & $\begin{array}{c}14.2_{-0.3}^{+0.3} \\
1.3_{-0.3}^{+0.3} \\
4.2_{-0.8}^{+0.9}\end{array}$ \\
\hline
\end{tabular}

${ }^{a}$ All errors were extracted at the $1 \sigma$ level.

b In all spectral fits, the column density was held fixed at the value found by Durant \& van Kerkwijk (2006b).

c All quoted fluxes are unabsorbed.

$\mathrm{d}$ The blackbody radius was calculated assuming a distance to the source of $3.5 \mathrm{kpc}$ (Durant \& van Kerkwijk 2006a).

e The width of this Gaussian line was not resolved, thus a Delta function was used smeared by the instrument response.

at the $>1-\sigma$ level for $\sim 130 \mathrm{~s}$. The significance of the $8.6 \mathrm{keV}$ feature is intermittent, but it remains detectable at the $>1-\sigma$ level beyond 130 s from the burst onset.

\subsection{Pulse Profile Changes}

Many AXP outbursts are accompanied by significant pulse profile changes (e.g. Kaspi et al. 2003). To search for these in $4 \mathrm{U} 0142+61$, for each observation, we generated 64-bin pulse profiles using the method described in Dib et al. (2007). We then aligned the profiles with a high signal-to-noise template using a cross-correlation procedure in the Fourier domain. Then, for each group of observations in the active phase, we summed the aligned profiles, extracted the DC component from the summed profile, and scaled the resulting profile so that the value of the highest bin is unity and the lowest bin is zero.

The resulting pulse profiles are shown in Figure 5 in chronological order. The panels in the left-most column are from Dib et al. (2007) and show pulse profiles in the 6 years prior to the entry into the active phase. Notice the slow change in the height of the dip between the peaks. The panels in columns 2 and 3, marked with the corresponding letter in the top right corner, show pulse profiles for each of the data groups in the active phase that were marked with a letter in Figure 1. The 4 plots in bold correspond to the observations marked with " $\mathrm{x}$ ", "b1", "b2", and "b3" in Figure 1

The pulse profile evolution can be described as follows: prior to the active phase, features in the double peaked pulse profile were evolving on a timescale of several years (see panels $\mathrm{C} 6$ to $\mathrm{C} 10$ corresponding to $R X T E$ Cycles 6 to 10). Then, in the first observation of the ac- tive phase (panel $\mathrm{x}$ ), the pulse profile became suddenly triple-peaked. It was also triple-peaked in the following observation (panel b1), in which a burst occurred. It remained multi-peaked for the following two groups of observations (panels $\mathrm{G}$ and $\mathrm{H}$ ). Then, an observation with multiple bursts occurred (panel b2). In that observation, also having a triple-peaked pulse profile, the middle peak was taller than the other two. Following this observation, the pulse profile seemed to be slowly recovering back to its double-peaked long-term shape (panels I, J, K, and L). Another burst observation interrupted this evolution seven months later (panel b3). In that observation, a large burst was detected. In the pulse profile of that observation, the left-most peak was significantly taller than usual. The event that caused this change had apparently no effect on the following observation which occurred 2 days later: the profile went back to being double-peaked (panels $\mathrm{M}$ and $\mathrm{N}$ ). To summarize, the pulse profile became multi-peaked at the beginning of the active phase. Following the second observation with bursts, the profile started to recover to its double-peaked shape. The evolution was only temporarily interrupted for the duration of the third observation with bursts. The behavior of the pulse profile in the $2-4$ and $4-10 \mathrm{keV}$ bands was similar.

Note that from Figure 5 alone we can compare the sizes of the peaks to each other, but we cannot track the evolution of the heights of each peak separately. In order to do that, we must scale the pulse profile of each group of observations by the average pulsed flux of that group. This analysis is presented in Section 3.4.3.

We also performed an analysis of the Fourier components of the pulse profiles. The results are shown in Figure 6. The variations in the power of the fundamental Fourier component are shown in panel 1, and that of the second harmonic in panel 2. Note how the amplitude of the fundamental varied monotonically prior to, but not during, the active phase. Also note how the power in the second harmonic was already back to its pre-active-phase level before the last burst occurred.

\subsection{Burst Rotational Phases}

An important factor in understanding the origin of the bursts is the rotational phase at which they occur. The phases of the bursts are shown in Figure 7 For each burst observation we created at 31.25-ms time resolution lightcurve and folded it using our timing solution (see $\S$ 3.5). We then phase-aligned these folded profiles by cross-correlating them with the long-term pulse profile template. Our phase-aligned folded profiles are shown in Figure 7 (histograms in the last row). Superposed on each folded profile are two curves. The top curve is made of the 5 Fourier harmonics that best fit the histogram. The bottom curve is made of the best-fit 5 harmonics after the removal of the 4 seconds centered on each burst. Note how the two curves in a given plot are similar, demonstrating that the additional peaks in the profiles are not due to the burst. In fact, for the brightest (and longest) burst, a fold of the last 1700 seconds of the data set shows that by the time the observation ended, the pulse profile had not recovered to its pre-burst shape.

The first three bursts occurred near the middle "new" peak in the profile. Burst 5 occurred near the old tall peak of the profile (see Fig. 5). The phase of burst 6 corresponds again to a new peak in the profile, this time 

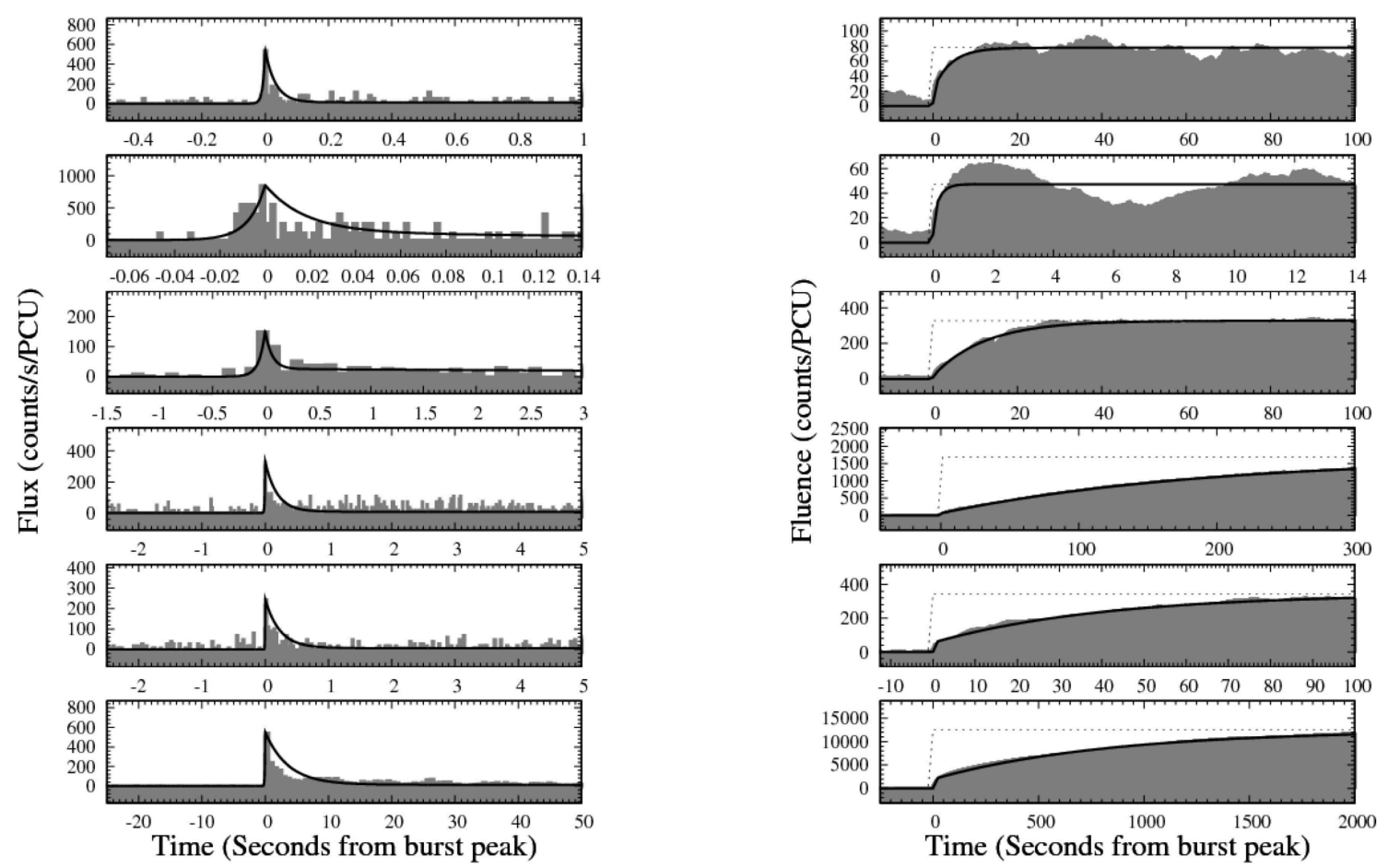

FIG. 2. - LEFT - The gray filled curves represent the 2-60 keV burst lightcurves as observed by $R X T E$. The binning was chosen such that the flux in the peak is equal to the model peak flux, $f_{p}$ (see Eq. 1), as determined by maximum likelihood likelihood testing. This binning scheme allow us resolve the bursts at the finest permissible time resolution. In descending order, the time bin widths are: $11.0,2.3$, 107.6, 18.3,32.6, and $686.3 \mathrm{~ms}$. The solid black curves are the best-fit exponential rise and decay models (Eq. 1). RIGHT - The gray filled curves represent the $2-60 \mathrm{keV}$ integrated burst lightcurves as observed by $R X T E$. For each burst lightcurve we subtracted the instrumental background, the background due to other sources in the FOV, and the contribution of the tails of neighboring bursts before integrating. Notice that some bursts have very long tails. The solid black curve is the integral of the model in the left panel. The dashed curve is a step function whose height corresponds to the total flux as determined by Eq. 2

located where the previous small peak used to be. The coincidences of several bursts with new, transient profile features that are present even when the actual burst data are removed are suggestive of lower-level transient emission from the same physical location, with the burst being the extreme of this emission's luminosity distribution.

\subsection{Pulsed Flux Analysis \\ 3.4.1. Short-Term Pulsed Flux Analysis}

Previous AXP bursts are often accompanied by shortterm pulsed flux enhancements (e.g. Gavriil et al. 2006). To search for these, for each of the three observations containing bursts, we made two time series in count rate per PCU, one for the $2-4 \mathrm{keV}$ band and the other for $4-20 \mathrm{keV}$. We included only photons detected by PCUs that were on for the entire duration of the observation. The time resolution was $31.25 \mathrm{~ms}$. We removed the $4 \mathrm{~s}$ centered on each burst from each time series. Then, we broke each time series into segments of length $\sim 500 \mathrm{~s}$. For each segment, we calculated the pulsed flux using two different methods.

First, we calculated the RMS pulsed flux using

$$
F_{\mathrm{RMS}}=\sqrt{2 \sum_{k=1}^{n}\left(\left(a_{k}^{2}+b_{k}^{2}\right)-\left({\sigma_{a_{k}}}^{2}+{\sigma_{b_{k}}}^{2}\right)\right)}
$$

where $a_{k}$ is the $k^{\text {th }}$ even Fourier component defined as
$a_{k}=\frac{1}{N} \sum_{i=1}^{N} p_{i} \cos (2 \pi k i / N), \sigma_{a_{k}}$ is the uncertainty of $a_{k}, b_{k}$ is the $k^{\text {th }}$ odd Fourier component defined as $b_{k}$ $=\frac{1}{N} \sum_{i=1}^{N} p_{i} \sin (2 \pi k i / N), \sigma_{b_{k}}$ is the uncertainty of $b_{k}, i$ refers to the phase bin, $N$ is the total number of phase bins, $p_{i}$ is the count rate in the $i^{\text {th }}$ phase bin of the pulse profile, and $n$ is the maximum number of Fourier harmonics to be taken into account; here $n=5$ for consistency with Dib et al. (2007) and Gonzalez et al. (2010).

While least sensitive to noise compared to other pulsed flux measurement methods, the RMS method returns a pulsed flux number that is affected by pulse profile changes. To confirm our pulsed flux results, we also used an area-based estimator to calculate the pulsed flux:

$$
F_{\text {Area }}=\frac{1}{N} \sum_{i=1}^{N} p_{i}-p_{\text {min }}
$$

where $p_{\min }$ is the average count rate in the off-pulse phase bins of the profile, determined by cross-correlating with a high signal-to-noise template, and calculated in the Fourier domain after truncating the Fourier series to 5 harmonics. The results are shown in Figure 8 for $F_{\mathrm{RMS}}$ ( $F_{\text {Area }}$ gives consistent results). Note the significant increase in the 4-20 keV pulsed flux in the 2006 June observation following the cluster of bursts. This increase is not present in $2-4 \mathrm{keV}$. Also note the significant rise and subsequent decay of the pulsed flux following the large 2007 February burst. The pulsed flux was sufficiently enhanced in these two observations that one can see in- 


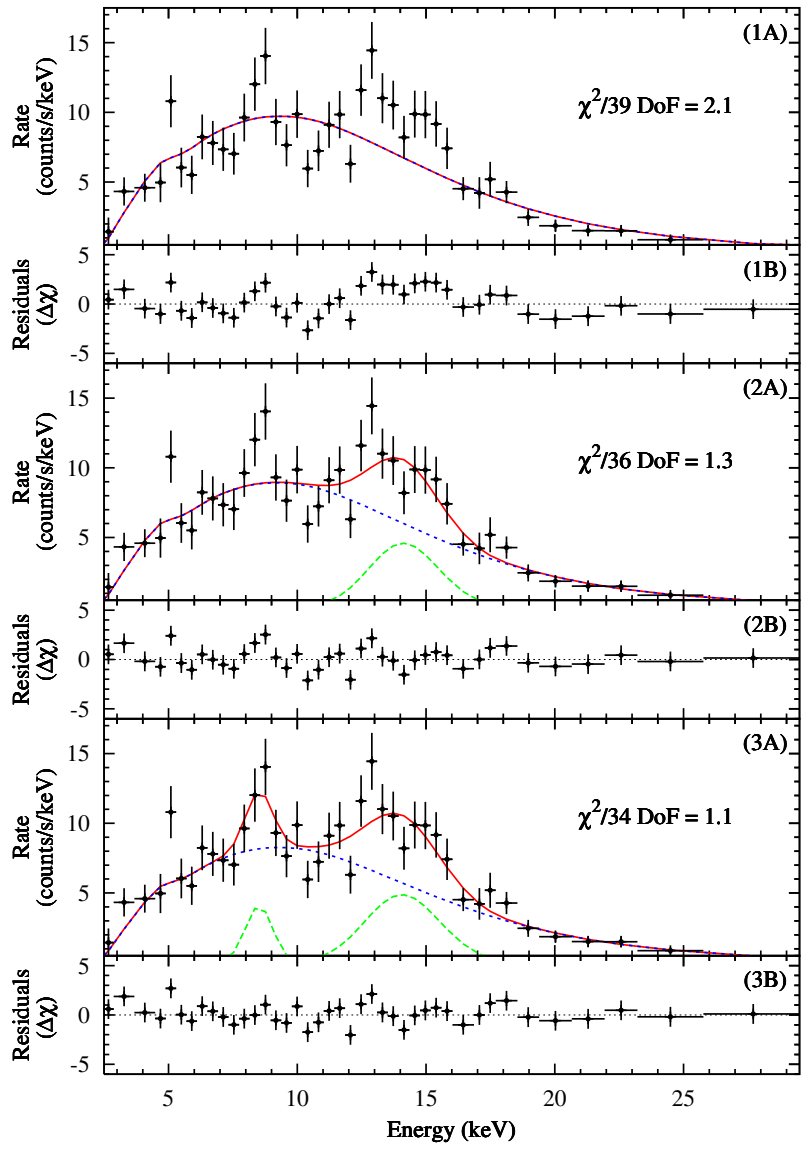

Fig. 3.- Panel 1A: The spectrum of burst 6 extracted over a pulse period $(8.69 \mathrm{~s})$ starting $50 \mathrm{~ms}$ before the burst peak. The red solid lines represents the best-fit model- a photoelectrically absorbed blackbody (red curve). The fit had a reduced $\chi^{2}$ of 2.1 for 39 degrees of freedom (DoF). Panel 1B: The difference between the data and the model shown in Panel 1A, divided by the uncertainty. Panels 2A and 2B : Same, except here the best-fit model (red curve) consists of a blackbody (dashed blue curve) plus a Gaussian emission lines (dashed green curve), all photoelectrically absorbed. The fit had a reduced $\chi^{2}$ of 1.3 for 36 DoF. Panels 3A and 3B: Same, except here the best-fit model (red curve) consists of a blackbody (blue curve) plus a two Gaussian emission lines (dashed green curves), all photoelectrically absorbed. The fit had a reduced $\chi^{2}$ of 1.1 for $34 \mathrm{DoF}$.

dividual pulsations by eye in Figure 7 in the two panels marked with a star, containing the raw burst lightcurves with 1-s time resolution.

\subsubsection{Long-Term Pulsed Flux Analysis}

For each of the 94 analyzed observations, we created a pulse profile (in units of count rate per PCU) using the same procedure as in Section 3.2. Then we calculated the pulsed flux for each observation using Equations 4 and 5. Data from PCU 0 were omitted because the longterm trend in the pulsed counts is not the same as that in the remaining PCUs, presumably due to the loss of its propane layer. Data from PCU 1 were omitted after the loss of its propane layer as well, on MJD 54094.

We extracted pulsed fluxes in the $2-4$ and $4-20 \mathrm{keV}$ bands using both the RMS and area pulsed flux method because of the numerous pulse profile changes around the times of the bursts. There is no evidence of a long-term change in the pulsed flux associated with the bursts except, possibly, for a hint of an increase in the $2-4 \mathrm{keV}$ band roughly midway between the second and third observations containing bursts. We also note that the 4-20 keV pulsed flux of two of the observations containing bursts are significantly larger than the long-term average. Removing the bursts from these observations does not change this result.

We performed the same analysis for individual observations in $2-10 \mathrm{keV}$ for an extended period of time. This is shown in panels 2 and 3 of Figure 9, This plot is an update to Figure 10 of Dib et al. (2007).

In order to verify that trends seen in panels 2 and 3 of Figure 9 are not an artifact of the evolution of the response of the detector, we calculated the pulsed flux in $\mathrm{erg} \mathrm{s}^{-1} \mathrm{~cm}^{-2}$ using a method that took the evolution of the response into account. The method is described in Dib et al. (2007) and takes into account spectral fits obtained from imaging data (in this case $X M M$ data, see Gonzalez et al. 2010) to convert counts to energy for each combined set of observations. For this analysis, we used data from all PCUs; however, data from PCU 1 were excluded after the loss its propane layer. Data from PCU 0 were included because the response matrices used took into account the loss of its propane layer.

The results are shown in panel 1 of Figure 9, The first 6 points, corresponding to $R X T E$ Cycles 6-10, are from Dib et al. (2007). The second to last point is obtained by combining all observations that occurred between bursts 1 and 2, but omitting observations containing bursts. The observations that we included took place during the exponential recovery of the possible glitch (see Section 3.5). The last point in panel 1 was obtained by combining the observations that occurred after burst 2, but again omitting those containing bursts. The observations we included took place after the end of the exponential recovery of the possible glitch (see Section 3.5).

In the first of the two data points in the active phase, the pulsed flux in erg s $\mathrm{s}^{-1} \mathrm{~cm}^{-2}$ is $18 \pm 8 \%$ larger than in the pre-active phase. This is consistent with the increase reported in Gonzalez et al. (2010) in the same energy range. A hint of this increase can be seen in panel 2 although it appears less significant. This discrepancy can be accounted for by the fact that the spectrum changed: Gonzalez et al. (2010) reported a temporary increase in the spectral hardness in an $X M M$ observation of $4 \mathrm{U} 0142+61$ immediately following the bursts.

\subsubsection{Combined Pulse Morphology and Pulsed Flux Analysis}

In Section 3.2, we calculated the Fourier components of the aligned average pulse profiles. This gave us the relative amplitude of the pulse profile harmonics in each group of observations marked with a letter in Figure 1. In Section 3.4.2, we calculated the pulsed flux for every observation. Here, we compute a weighted average of the pulsed flux for each group of observations using the flux points calculated in Section 3.4.2 We then reconstruct the profiles for each of the groups from the first five Fourier components (not including the DC), scale them by the average RMS pulsed flux for that group, and add the necessary offset for the lowest point on each curve to be zero. This means that the resulting scaled profiles return the correct pulsed flux. The advantage of this analysis is that we can now trace the evolution of 


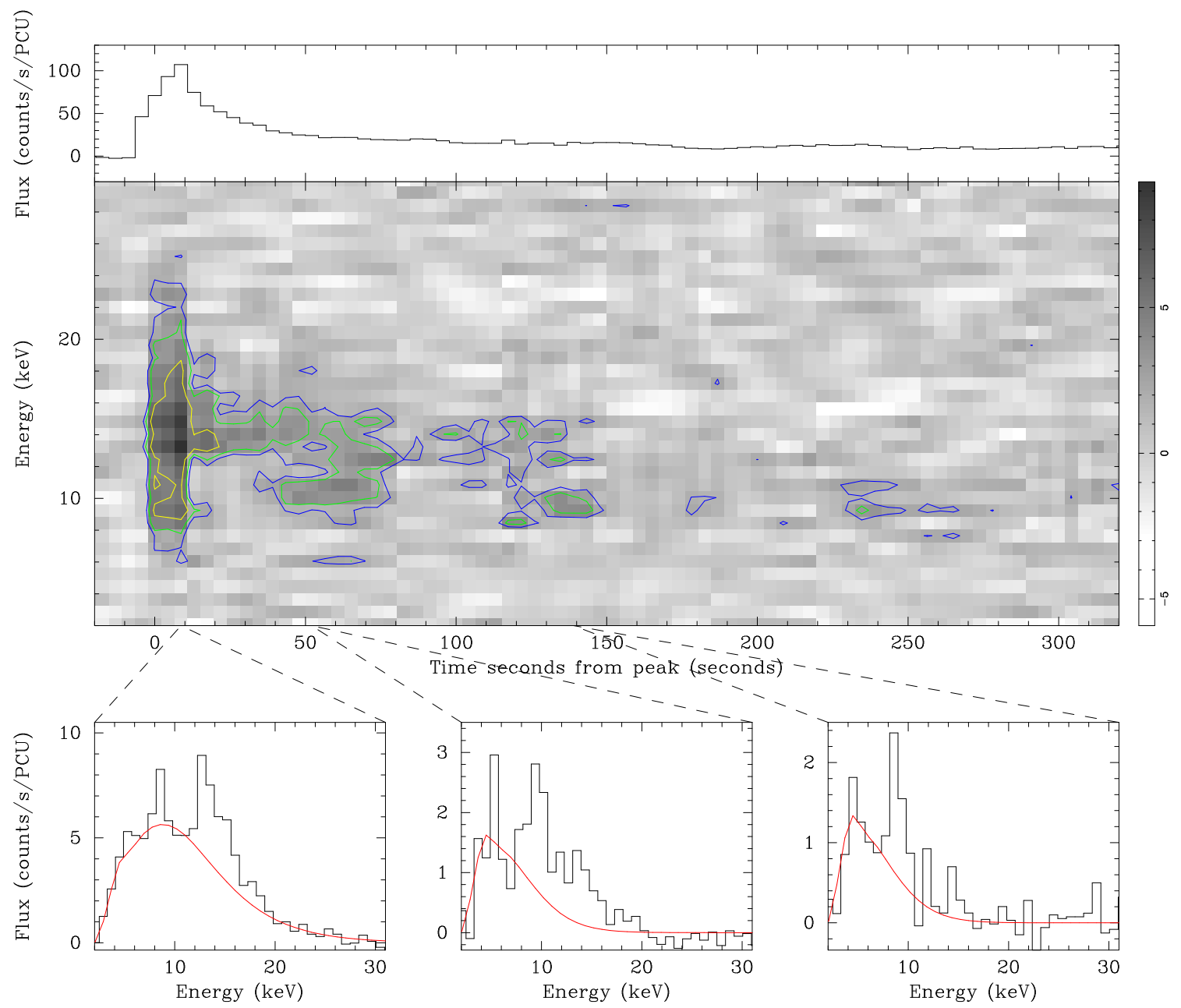

FIG. 4.- Spectral evolution of burst 6. Top: Burst lightcurve binned with 4.34-s (half a pulse period) time resolution and extracted over the same energy band used for spectral fitting, 2.5-30 keV. Middle: Surface plot of the difference between the burst spectrum and a simple continuum model (blackbody) in terms of change in $\chi^{2}$. Each vertical slice in the surface plot corresponds to a spectrum that was calculated over a two-pulse-period long window. This window was translated across the burst in half-a-pulse-period long steps. For each spectrum, a best-fit blackbody model was subtracted. The color wedge on the right maps the colors to the resulting change in $\chi$. The contours indicate the regions where the spectrum differed by the model by $1-\sigma$ (blue contour), $2-\sigma$ (green contour) and 3- $\sigma$ (yellow contour), respectively. The feature at $14.2 \mathrm{keV}$ is highly significant at the start of the burst, and remains detectable at the $>1-\sigma$ level for $\sim 130 \mathrm{~s}$. Notice, that the significance of the $8.6 \mathrm{keV}$ feature is intermittent, becoming comparable to that of the $14.2 \mathrm{keV}$ feature at later times, and is the only feature detectable at the $>1-\sigma$ beyond $\sim 130$ s from the burst peak. Bottom: The insets are examples of individual two-pulse-period long spectra used to generate the surface plot. These spectra were extracted at 8.69, 52.1 and $139.0 \mathrm{~s}$ from the burst onset, respectively. The red curves indicate the corresponding best-fit blackbody model.

each of the peaks independently.

The results are presented in Figure10. In panel (a), we show the average scaled pulse profile per observation for observations in the year preceding the active phase. The profile is double-peaked. In panel (b), we show in bold the scaled profile for the observation containing burst 1 . We also show the scaled profiles in each of the groups of observations that followed the burst. The profiles are triple-peaked. In panel (c), we show in bold the scaled profile for the observation containing bursts $2,3,4$, and 5 . We also show the scaled profiles in each of the groups of observations that followed the bursts. Note how the increase in the pulsed flux in the observation containing the bursts is not only a consequence of the appearance of the new peak, but a result of the increase in size of all three peaks. Also note the evolution of the pulse profile back to being double-peaked. In panel (d), we show in bold the scaled profile for the observation containing burst 6 . We also show the scaled profiles in each of the groups of observations that followed the burst. Note again how the pulsed flux increase is due to both peaks increasing in size.

\subsection{Timing Analysis}

Many AXP outbursts and active phases are accompanied by interesting timing anomalies (e.g. Kaspi et al. 2003; Woods et al. 2004; Israel et al. 2007a; Dib et al. 2009). Next we consider the timing behavior of $4 \mathrm{U} 0142+61$, which, as we show, also exhibits interesting evolution at the start of the active phase.

For all our $R X T E$ observations of $4 \mathrm{U} 0142+61$ we created $31.25-\mathrm{ms}$ time resolution lightcurves, including only the events in the energy range $2.5-9 \mathrm{keV}$ to maximize the signal-to-noise ratio of the pulse. Each binned time series was epoch-folded using an ephemeris determined iteratively by maintaining phase coherence; see below. Resulting pulse profiles, with 64 phase bins, were crosscorrelated in the Fourier domain with a high signal-to- 

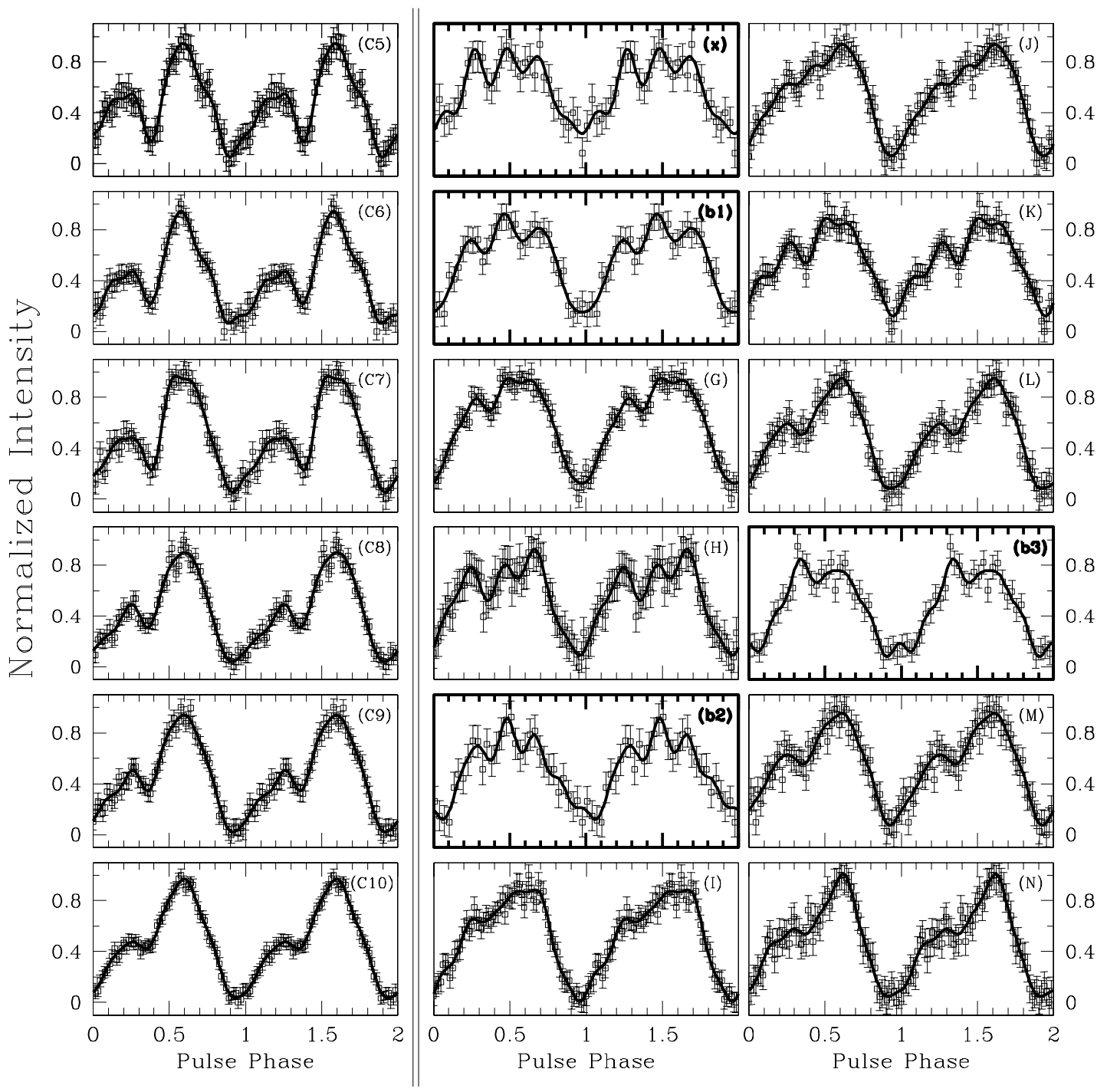

FIG. 5.- Pulse profile evolution of $4 \mathrm{U} 0142+61$. Left-most column: normalized average $2-10 \mathrm{keV}$ pulse profiles in the 6 years prior to the entry into the active phase in chronological order (panels from Dib et al. |2007). Middle and right-most columns: normalized, averaged $2-10 \mathrm{keV}$ pulse profiles for each of the data groups marked by a letter in Figure 1 after the entry into the active phase. The 4 plots with bold labels correspond to the observations marked with "x", "b1", "b2", and "b3" in Figure 1

noise template created by adding phase-aligned profiles from all observations. The cross-correlation returned an average pulse time of arrival (TOA) for each observation corresponding to a fixed pulse phase. The pulse phase $\phi$ at any time $t$ can be expressed as a Taylor expansion,

$$
\begin{aligned}
\phi(t)= & \phi_{0}\left(t_{0}\right)+\nu_{0}\left(t-t_{0}\right)+\frac{1}{2} \dot{\nu_{0}}\left(t-t_{0}\right)^{2} \\
& +\frac{1}{6} \ddot{\nu_{0}}\left(t-t_{0}\right)^{3}+\ldots,
\end{aligned}
$$

where $\nu \equiv 1 / P$ is the pulse frequency, $\dot{\nu} \equiv d \nu / d t$, etc., and subscript " 0 " denotes a parameter evaluated at the reference epoch $t=t_{0}$. To obtain ephemerides, the TOAs were fitted to the above polynomial using the pulsar timing software package TEMPO].

As explained above, our first set of TOAs was obtained

\footnotetext{
${ }^{7}$ See http://www.atnf.csiro.au/research/pulsar/tempo
}

by aligning the folded observations with a template profile using a cross-correlation procedure. In order to determine to what extent this set of TOAs was affected by the pulse profile changes that took place in the active phase, we generated two additional sets of TOAs (sets 2 and 3 ).

For set 2, we made the assumption that the location of the trough of the pulsations, determined by finding the minimum in the pulse profile after smoothing, is not affected by the pulse profile changes. We then generated TOAs by aligning the pulsation trough of the folded observations with that of the template and extracting phase differences. The last set of TOAs (Set 3) was obtained after aligning the tallest peak of each smoothed pulse profile with that of the long-term template. All three sets of TOAs are provided as a machine readable table in the electronic edition. A portion of the table starting from the active phase is provided in Table 3 ,

Timing residuals for all three sets of TOAs are shown 


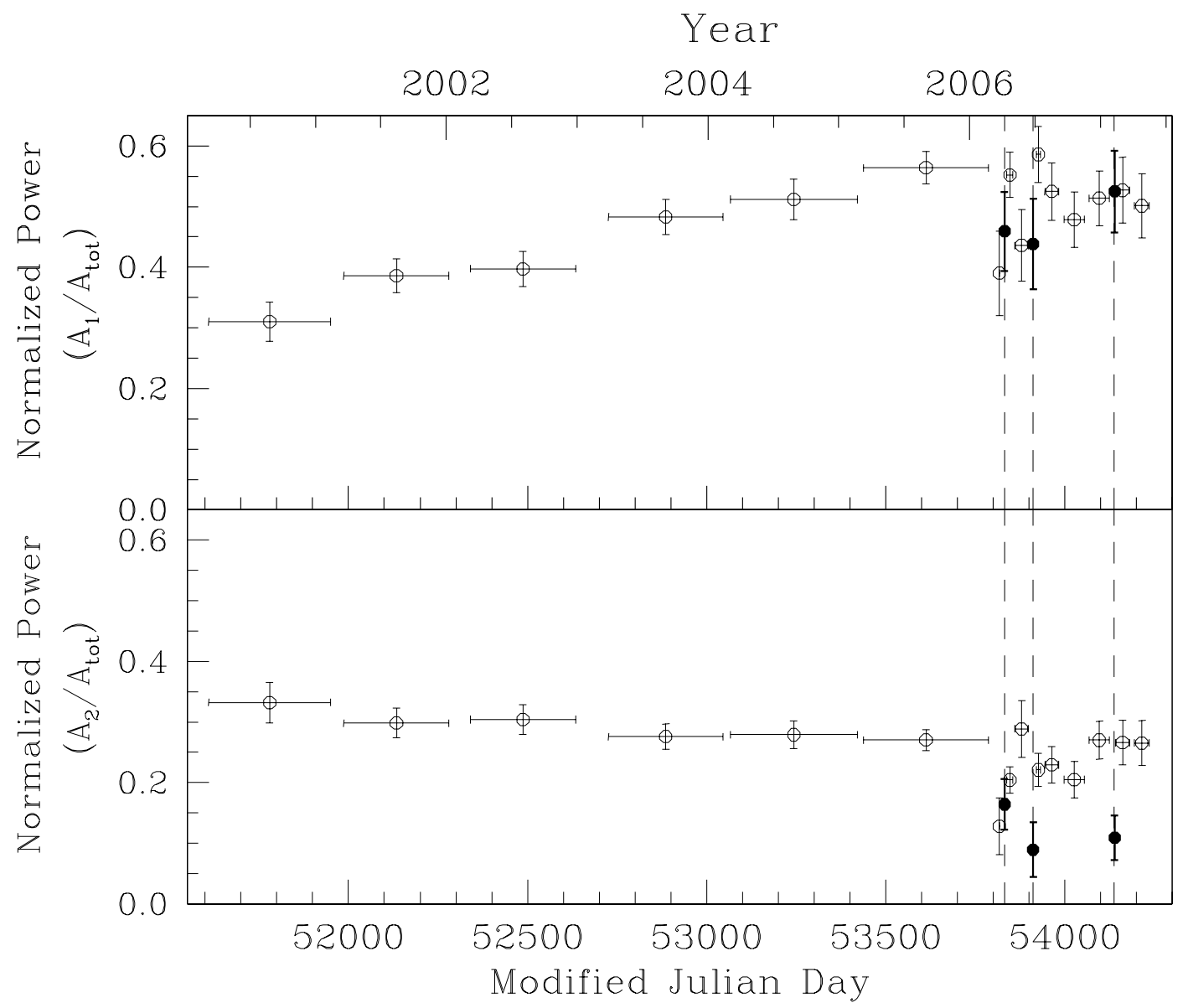

FIG. 6. - Top: evolution of the power in the fundamental Fourier component of the pulse profile of $4 \mathrm{U} 0142+61$ in the $2-10 \mathrm{keV}$ band. Bottom: Evolution of the power in the second harmonic. In both panels, the points with large horizontal error bars are from Dib et al. (2007). The remaining points are obtained from groups of observations after the entry into the active phase. The three bold points correspond to the observations with bursts. The three dashed lines correspond to burst epochs.

TABLE 3

A Sample of our RXTE timing observation log for 4 U $0142+61$. The full Version of this table is provided in the ELECTRONIC EDITION OF THE JOURNAL.

\begin{tabular}{|c|c|c|c|c|c|c|c|c|c|c|}
\hline \multirow[b]{2}{*}{$\begin{array}{c}\text { TOA } \\
\text { Numb. }^{\text {a }}\end{array}$} & \multirow[b]{2}{*}{ Obs. ID $^{\mathrm{b}}$} & \multirow[b]{2}{*}{$\begin{array}{c}\text { Exp. }{ }^{\mathrm{c}} \\
(\mathrm{ks})\end{array}$} & \multirow[b]{2}{*}{$\begin{array}{l}\text { Active } \\
\text { PCUs }^{d}\end{array}$} & \multicolumn{2}{|l|}{ Set 1} & \multicolumn{2}{|l|}{ Set 2} & \multicolumn{2}{|l|}{ Set 3} & \multirow[b]{2}{*}{$\begin{array}{c}\mathrm{PT} \\
\text { Analysis? }\end{array}$} \\
\hline & & & & $\begin{array}{l}\mathrm{TOA}^{\mathrm{e}} \\
(\mathrm{MJD})\end{array}$ & $\begin{array}{c}\bar{\sigma}_{\mathrm{TOA}}{ }^{\mathrm{f}} \\
(\mathrm{s})\end{array}$ & $\begin{array}{l}\mathrm{TOA}^{\mathrm{e}} \\
(\mathrm{MJD})\end{array}$ & $\begin{array}{c}\bar{\sigma}_{\mathrm{TOA}}^{\mathrm{f}} \\
(\mathrm{s})\end{array}$ & $\begin{array}{l}\overline{\mathrm{TOA}^{\mathrm{e}}} \\
(\mathrm{MJD})\end{array}$ & $\begin{array}{c}\bar{\sigma}_{\mathrm{TOA}}^{\mathrm{f}} \\
(\mathrm{s})\end{array}$ & \\
\hline 33 & $92006-05-02-00$ & 4.9 & 3 & 53817.1615364 & 0.08 & 53817.1616238 & 0.20 & 53817.1615403 & 0.20 & Yes \\
\hline 34 & $92006-05-03-00$ & 4.5 & 3 & 53831.3358686 & 0.09 & 53831.3358558 & 0.21 & 53831.3358711 & 0.21 & Yes \\
\hline 58 & 92006-05-09-01 & 1.0 & 3 & 53911.0530210 & 0.17 & 53911.0531137 & 0.43 & 53911.0530298 & 0.43 & Yes \\
\hline 78 & $92006-05-25-00$ & 5.3 & 2 & 54138.4487229 & 0.11 & 54138.4487002 & 0.29 & 54138.4487277 & 0.29 & No \\
\hline
\end{tabular}

a TOA number. TOA number 33 is the first observation in the active phase. TOAs number 34,58 , and 78 represent the first, second and third observation containing bursts.

b RXTE observation identification number.

c The effective number of PCUs on during the observation.

d Total exposure.

e Barycentered pulse TOA. Set corresponds to the TOAs obtained by cross-correlating the folded pulse profiles with a high signalto-noise template. Sets 2 and 3 are similar to Set 1 , however, we constrained our cross-correlation to the trough and peak of the pulsations, respectively. See $\S 3.5$ for details.

${ }^{\mathrm{f}}$ Uncertainty on TOA for Set 1,2 , and 3 .

$\mathrm{g}$ Whether the TOA was included in the partial timing analysis. Note: All TOAs were included in the long-term analysis. 

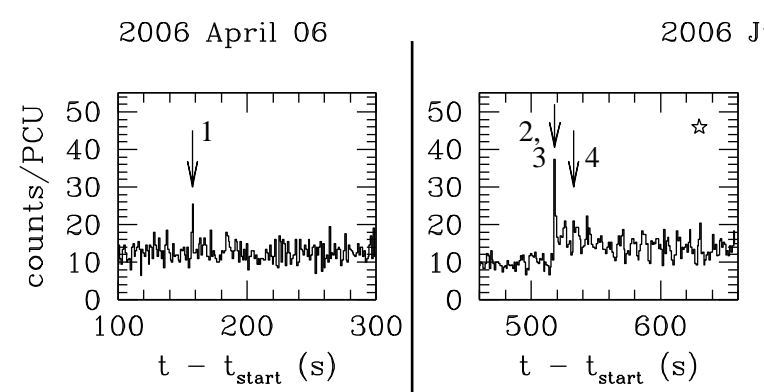

2006 June 25

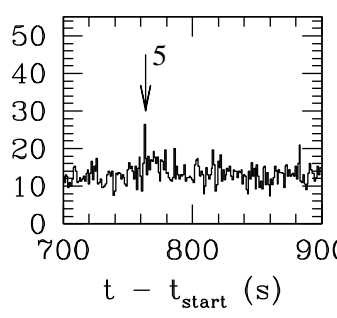

2007 February 07
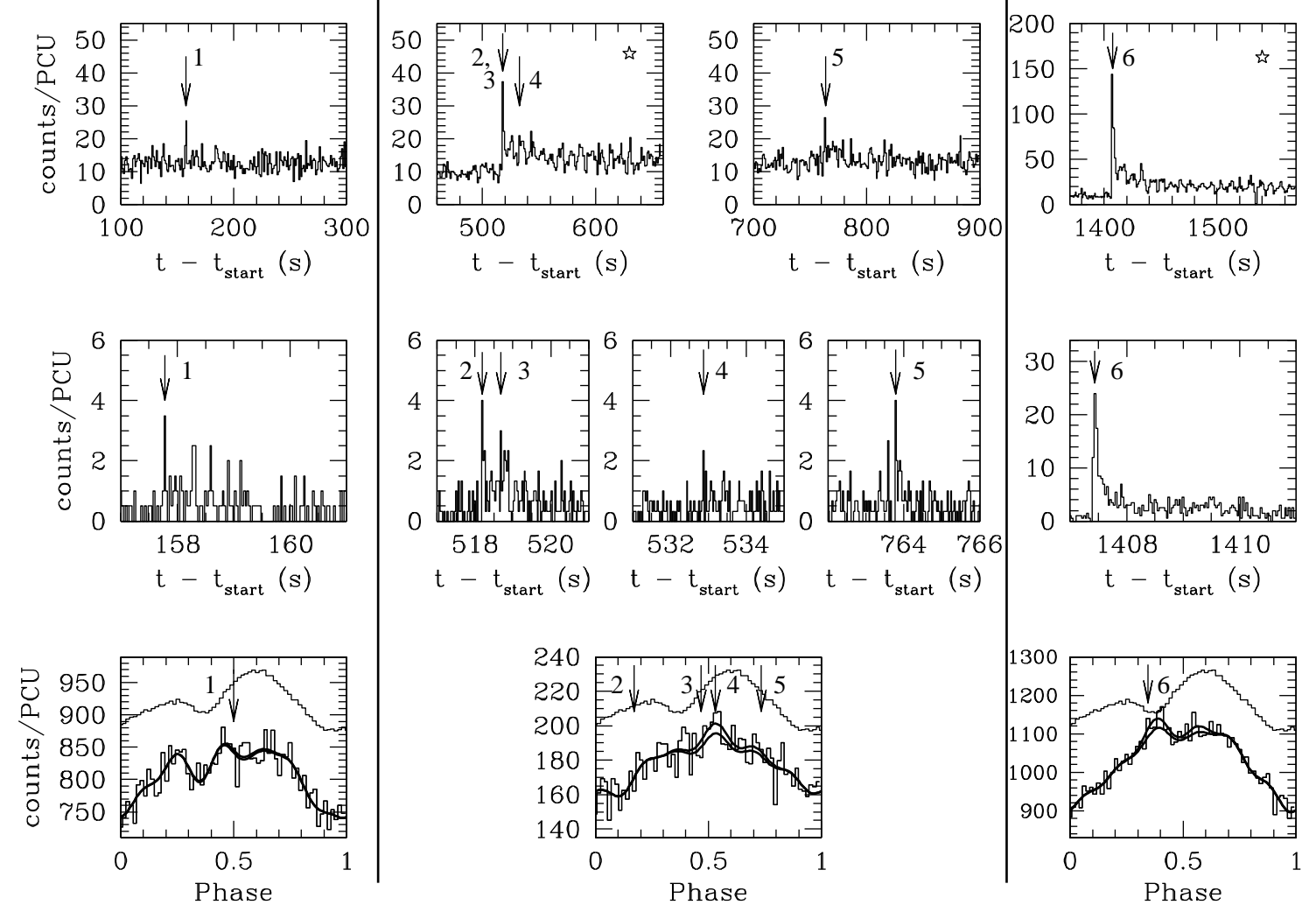

FIG. 7.- Phase analysis of the 6 detected bursts. Each column corresponds to an observation in which bursts were detected. Top row: 200-s segments of the time series containing the bursts in the $2-20 \mathrm{keV}$ band. The time resolution is 1 s. Middle row: 4 -s segments of the time series containing the bursts. The time resolution $31.25 \mathrm{~ms}$. Bottom row: aligned folds of the burst observations shown below the scaled long-term average profile. The folded counts are presented as histograms. Superposed on each histogram are two curves. The top curve is made of the 5 Fourier harmonics that best fit the histogram. The bottom curve is made of the best-fit 5 harmonics after the removal of the 4 seconds centered on each burst. The arrows indicate the phases of each burst. 

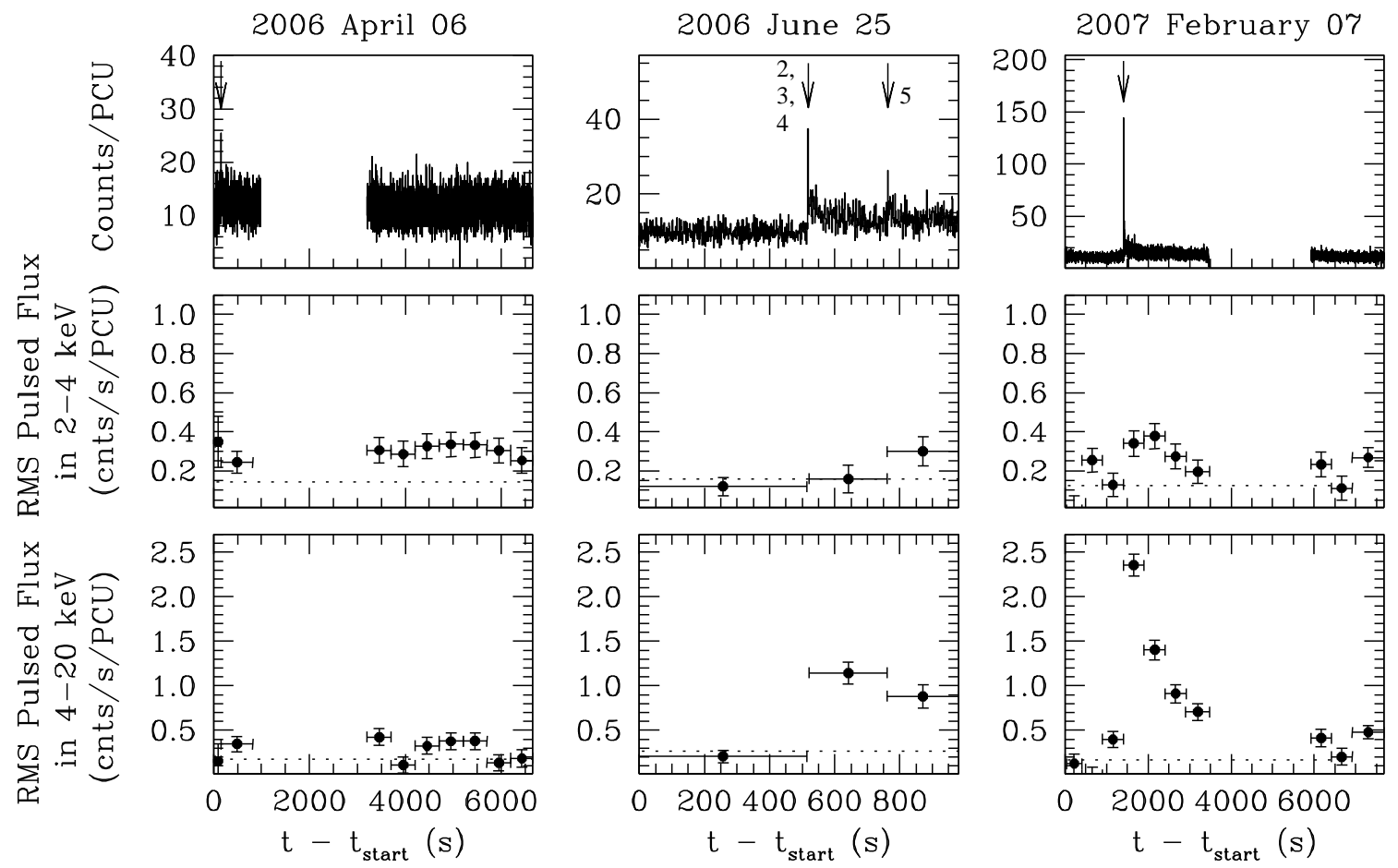

FIG. 8. - RMS pulsed flux within the observations containing bursts. Each column corresponds to one observation. In each column we show, descending vertically, the 1-s resolution lightcurve with the bursts indicated, the 2-4 keV RMS pulsed flux, and the 4-20 keV RMS pulsed flux. The dotted line in each of the pulsed flux plots shows the average of the pulsed fluxes obtained after segmenting and analyzing the time series of the observation immediately prior to the one shown.

in Figure 11 using two different ephemerides. The linear ephemeris used in the top panel is the ephemeris that best fit the first set of TOAs prior to the active phase. The linear ephemeris used in the bottom panel is that obtained by fitting the first set of TOAs in the regions indicated by the horizontal arrows, with a phase jump in between. The same long-term trend is present in the first two sets of timing residuals, with more scatter in the second set. The difference in phase between each bold circle and the corresponding empty circle represents our uncertainty in determining a fiducial point on the pulsar. In the third set of residuals, the outlier points represent the observations where the largest peak was no longer the right-most peak. Apart from the outliers, the same trend seen in the other two sets of residuals is present in the third set.

From here on, we assume that the presence of the same trend in our three sets of residuals is an indication that the TOAs in the first set were not significantly affected by the pulse profile changes. We therefore have used the first set of TOAs (one for each of the 94 observations) in the remainder of this Section.

Using the above assumption, the results of the timing analysis are shown in Figure 12, The results of a segmented timing analysis are in panels 1 and 2 . The results of a long-term timing analysis are in panels 3,4 , 5 , and 6 of the same Figure.

For the segmented timing analysis, we used the 70 TOAs that had the smallest uncertainties (indicated in the online version of Table 3) and omitted the TOAs of the 3 burst observations as well as the TOA of the first observation in the active phase. We then divided the data into segments of similar pulse profiles. For each data segment we found a linear ephemeris using TEMPO 8 . We plotted the resulting ephemerides in panel 1 of Figure 12 with the uncertainties returned by TEMPO. The timing residuals are shown in panel 2 . Notice how the slope between bursts 1 and 2 is more negative than the long-term average.

We then fit a linear trend through all the pre-burst observations, and another linear ephemeris through the post-burst observations after the pulse profile had started to return to the double-peaked shape. We did not include the TOA corresponding to the last observation containing bursts. The results are plotted in panel 3 of Figure 12 with uncertainties, with the residuals in panel 4 . Notice the difference in the slope in the two regions. In particular, extrapolating the two ephemerides to a point between their times of validity makes it seem like a sudden spin down, i.e. an "anti-glitch" occurred.

Finally, we included all the TOAs and did one global fit. In order to provide a good fit to the TOAs at the onset of the active phase, we had to assume that a glitch occurred on MJD 53809, with the glitch model consisting of a permanent change in $\nu$ and $\dot{\nu}$ and a frequency change $\Delta \nu_{d}$ that recovered exponentially on a timescale $\tau_{d}$, i.e.,

$$
\nu=\nu_{0}(t)+\Delta \nu+\Delta \nu_{d} e^{-\left(t-t_{g}\right) / \tau_{d}}+\Delta \dot{\nu}\left(t-t_{g}\right),
$$

where $\nu_{0}(t)$ is the frequency evolution pre-glitch given by $\nu_{0}(t)=\nu\left(t_{0}\right)+\dot{\nu}\left(t-t_{0}\right), \Delta \nu$ is a instantaneous unrecovered frequency jump, $\Delta \nu_{d}$ is the frequency increase that decays exponentially on a time scale $\tau_{d}, t_{g}$ is the glitch epoch determined by setting the phase jump to zero, and $\Delta \dot{\nu}$ is the post-glitch change in the long-term

\footnotetext{
${ }^{8}$ For the segmented timing analysis we ran TEMPO in mode 1.
} 


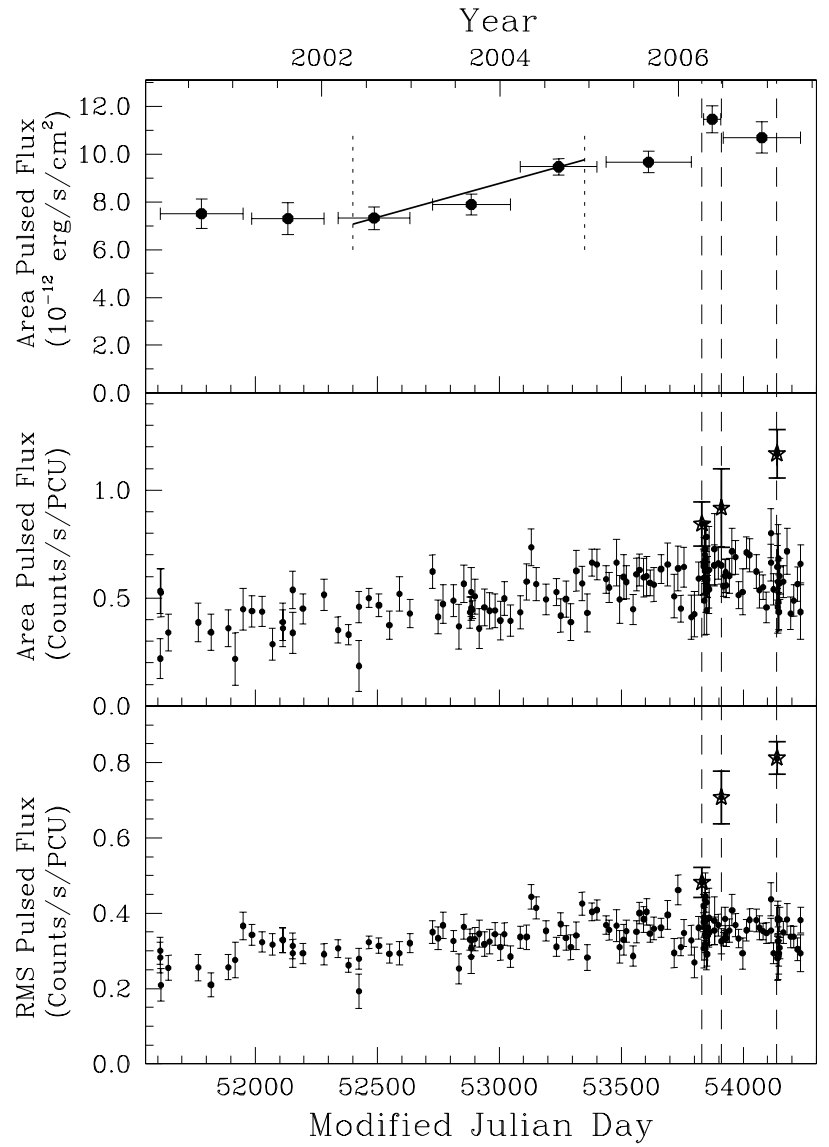

FIG. 9.- Long-term pulsed flux time series in $2-10 \mathrm{keV}$ for $4 \mathrm{U}$ 0142+61. Panel 1: area pulsed flux in erg $\mathrm{s}^{-1} \mathrm{~cm}^{-2}$ for combined observations. The solid line marks the $29 \pm 8 \%$ increase reported in Dib et al. (2007)). Panel 2: area pulsed flux in counts/s/PCU for individual observations. Panel 3: RMS pulsed flux in counts/s/PCU for individual observations. All panels: the dashed lines mark the burst epochs. The points marked with stars are the pulsed fluxes of the observations containing bursts.

frequency derivative. The values of the fit parameters are listed in Table 4 .

From Table 4, we can see that the total sudden change in frequency which happened at the onset of the active phase $\left(\Delta \nu_{t o t}=\Delta \nu+\Delta \nu_{d}\right)$ is positive, and $\Delta \nu_{d}$ decayed exponentially. At the end of the decay, the net effect of the recovered glitch was a negative $\Delta \nu$. That is, the data suggest that after $\nu_{d}$ had decayed, there remained net spin-down relative to the undisturbed ephemeris from before the active phase.

The fit parameter $\Delta \nu_{d}$, from which we conclude a sudden spin-up, is controlled primarily by the first few TOAs in the active phase. It is therefore possible that it is affected by pulse profile changes. However, as shown in Figure 11, the TOAs in the active phase were not significantly contaminated by pulse profile changes. Also, the segmented analysis shown in Figure 12 clearly indicates an initial spin-up.

In contrast to $\Delta \nu_{d}$, the fit parameter $\Delta \nu$, from which we conclude a net spin down, is primarily controlled by the TOAs outside of the active phase, which were certainly not affected by pulse profile changes (Figure 12 panel 3).

We therefore conclude that the pulsar likely suffered a

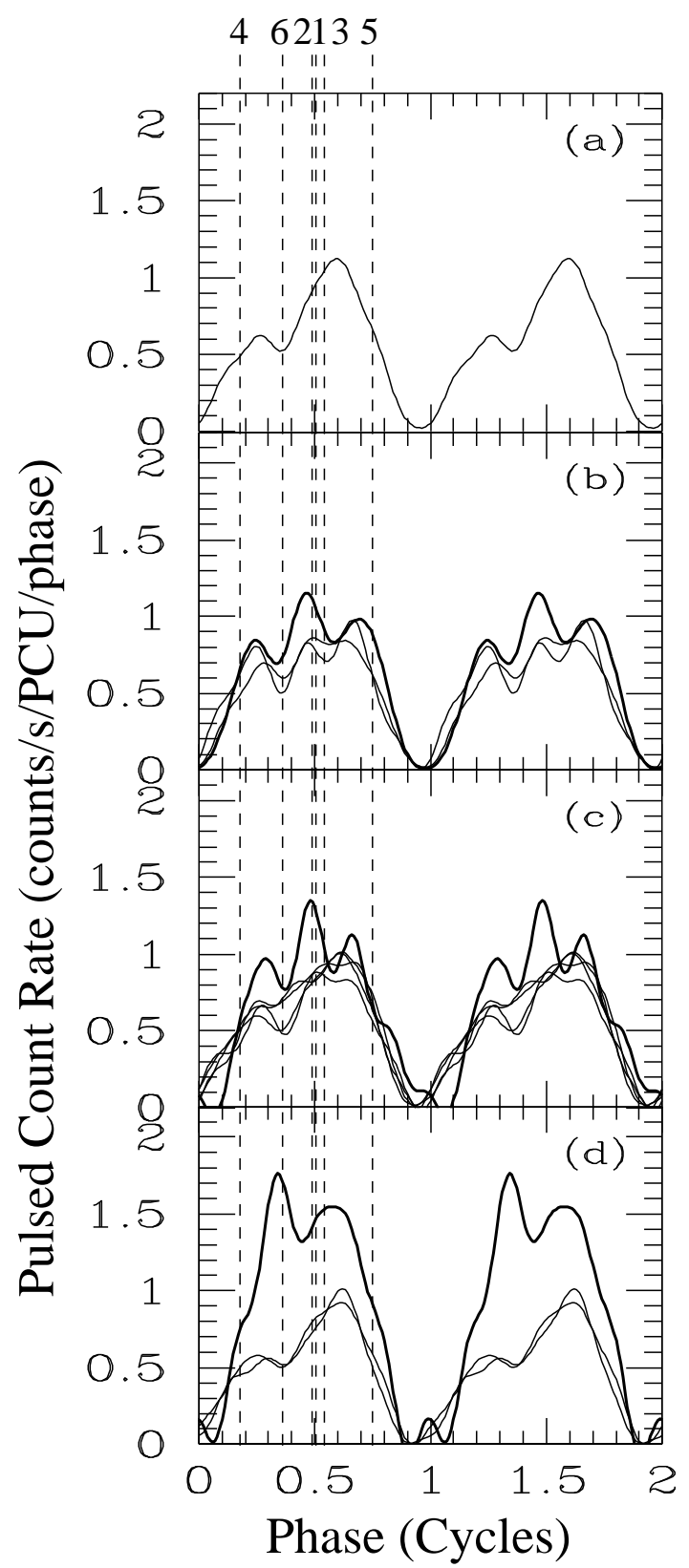

FIG. 10.- Average pulse profile per observation in the $2-10 \mathrm{keV}$ band in several groups of observations reconstructed from the first five Fourier components, and scaled to return the appropriate pulsed flux. (a) average scaled pulse profile for observations in $R X T E$ Cycle 10, the last RXTE Cycle before the active phase. (b) Bold curve: scaled pulse profile of the observation containing burst 1. Thin curves: average scaled pulse profile for each of the groups of observations that followed burst 1 (groups $\mathrm{G}$ and $\mathrm{H}$ in Figure 11). (c) Bold curve: scaled pulse profile of the observation containing bursts $2,3,4$, and 5 . Thin curves: average scaled pulse profile for each of the groups of observations that followed the bursts (groups I, J, K, and L in Figure 1). (d) Bold curve: scaled pulse profile of the observation containing burst 6 . Thin curves: average scaled pulse profile for each of the groups of observations that followed burst 6 (groups $\mathrm{M}$, and N from Figure 1). The vertical dotted lines indicate the phases of each burst. 


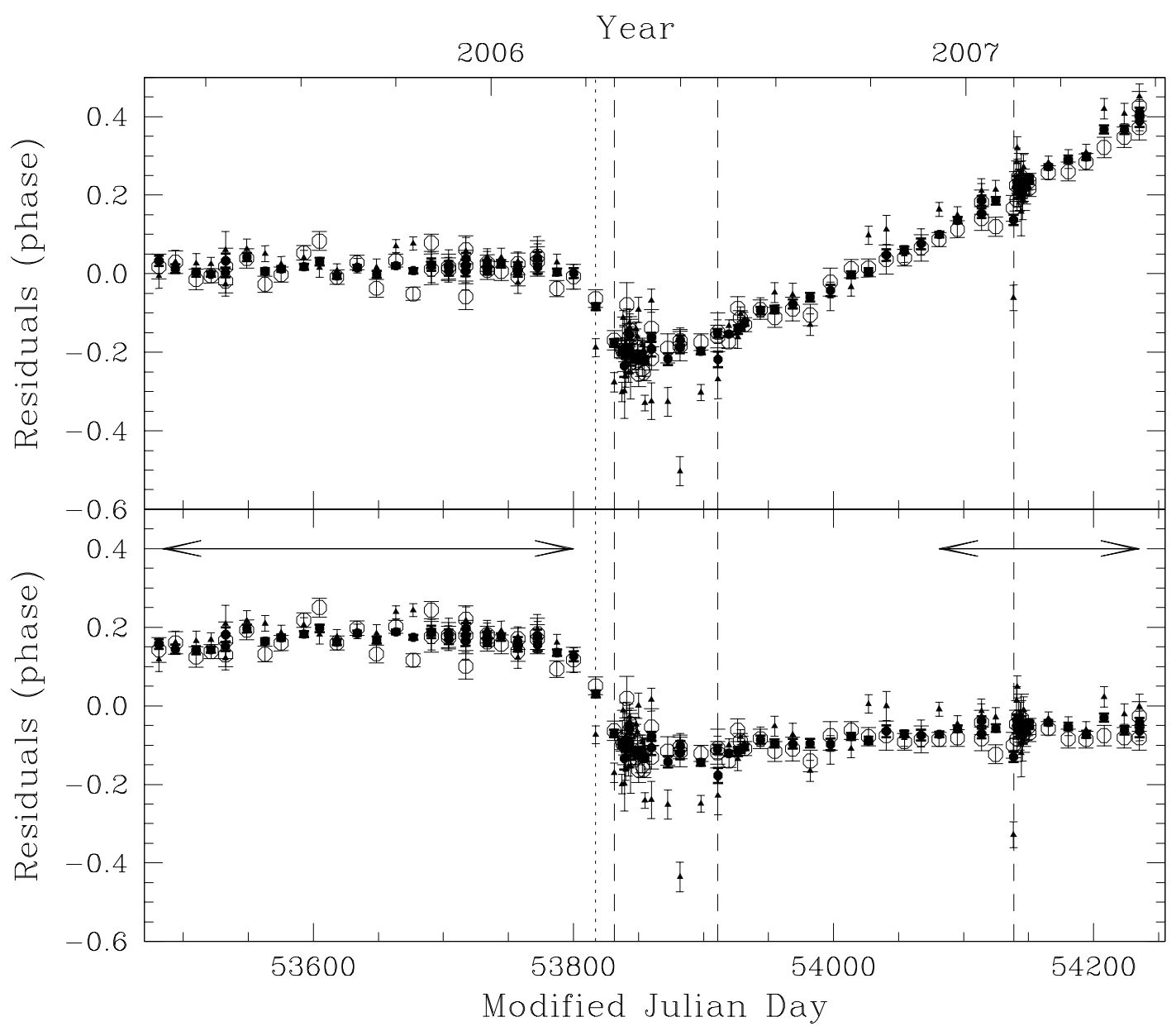

FIG. 11. - Top: Timing residuals for all three sets of TOAs. Bold circles: timing residuals obtained using the first set of TOAs obtained by cross correlation in the Fourier domain. Empty circles: residuals obtained using the second set of TOAs obtained by aligning the off-pulse regions of the pulse profiles. Triangles: residuals obtained using the third set of TOAs obtained by aligning the tallest peak of the pulse profiles. The linear ephemeris used to produce all three sets of residuals is that which best fit the first set of TOAs in the pre-active phase region. Bottom: Timing residuals for the same three sets of TOAs. The linear ephemeris used is that which best fit the first set of TOAs exclusively in regions indicated with the horizontal arrows with an arbitrary phase jump in the gap between the arrows. The phase residuals are relative to the linear ephemeris (ignoring the phase jump) and vertically offset such that the average phase residual is zero. Both panels: the dotted line indicates the epoch of the first observation of the active phase. The dashed lines indicate the burst epochs.

TABLE 4

Spin and Glitch PARAmeters For $4 \mathrm{U} 0142+61^{\mathrm{a}}$

\begin{tabular}{lc}
\hline \hline \multicolumn{1}{c}{ Parameter } & Value \\
\hline MJD start & 53481.268 \\
MJD end & 54235.319 \\
TOAs & $93^{\mathrm{b}}$ \\
$\nu(\mathrm{Hz})$ & $0.1150920955(12)$ \\
$\dot{\nu}\left(10^{-14} \mathrm{~Hz} \mathrm{~s}^{-1}\right)$ & $-2.661(9)$ \\
Epoch $(\mathrm{MJD})$ & 53809.185840 \\
Glitch Epoch (MJD) & 53809.185840 \\
$\Delta \nu(\mathrm{Hz})$ & $-1.27(17) \times 10^{-8}$ \\
$\Delta \dot{\nu}\left(\mathrm{Hz} \mathrm{s}{ }^{-1}\right)$ & $-3.1(1.2) \times 10^{-16}$ \\
$\Delta \nu_{d}(\mathrm{~Hz})$ & $2.0(4) \times 10^{-7}$ \\
$t_{d}$ (days) & $17.0(1.7)$ \\
RMS residual (phase) & 0.0168 \\
\hline
\end{tabular}

${ }^{a}$ Numbers in parentheses are TEMPO-reported $1 \sigma$ uncertainties. b A single TOA was omitted due to the very poor signal to noise ratio in the corresponding observation.

spin-up glitch near or at the start of the radiatively active phase, but that the glitch 'over-recovered' such that longterm, its net effect is a spin-down of the pulsar.

\section{DISCUSSION}

In this paper, we have described the timing, pulse profile, and pulsed flux behavior of $4 \mathrm{U} 0142+61$, during its 2006-2007 active phase, the first such episode yet studied from this source. Specifically we have shown that in addition to a sudden departure from a slow, systematic evolution of the source's pulsed flux and pulse profile, this AXP also suffered a significant timing event that is best described as a sudden spin-up glitch, followed by a large decay of the frequency jump such that the net effect was a slow-down with $\Delta \nu / \nu \simeq-8 \times 10^{-8}$. Interestingly, the pulsed X-ray flux showed no significant change apart from immediately following bursts, in contrast to other AXP radiative outbursts (e.g. Kaspi et al. 2003; Tam et al. 2008; Ibrahim et al. 2004; Muno et al. 2007). Further, we have reported on six SGR-like bursts from the source that occurred during this period, one of which was notable for its unusual spectrum, which was poorly fit by an continuum model.

\subsection{The SGR-Like Bursts from $4 U 0142+61$}



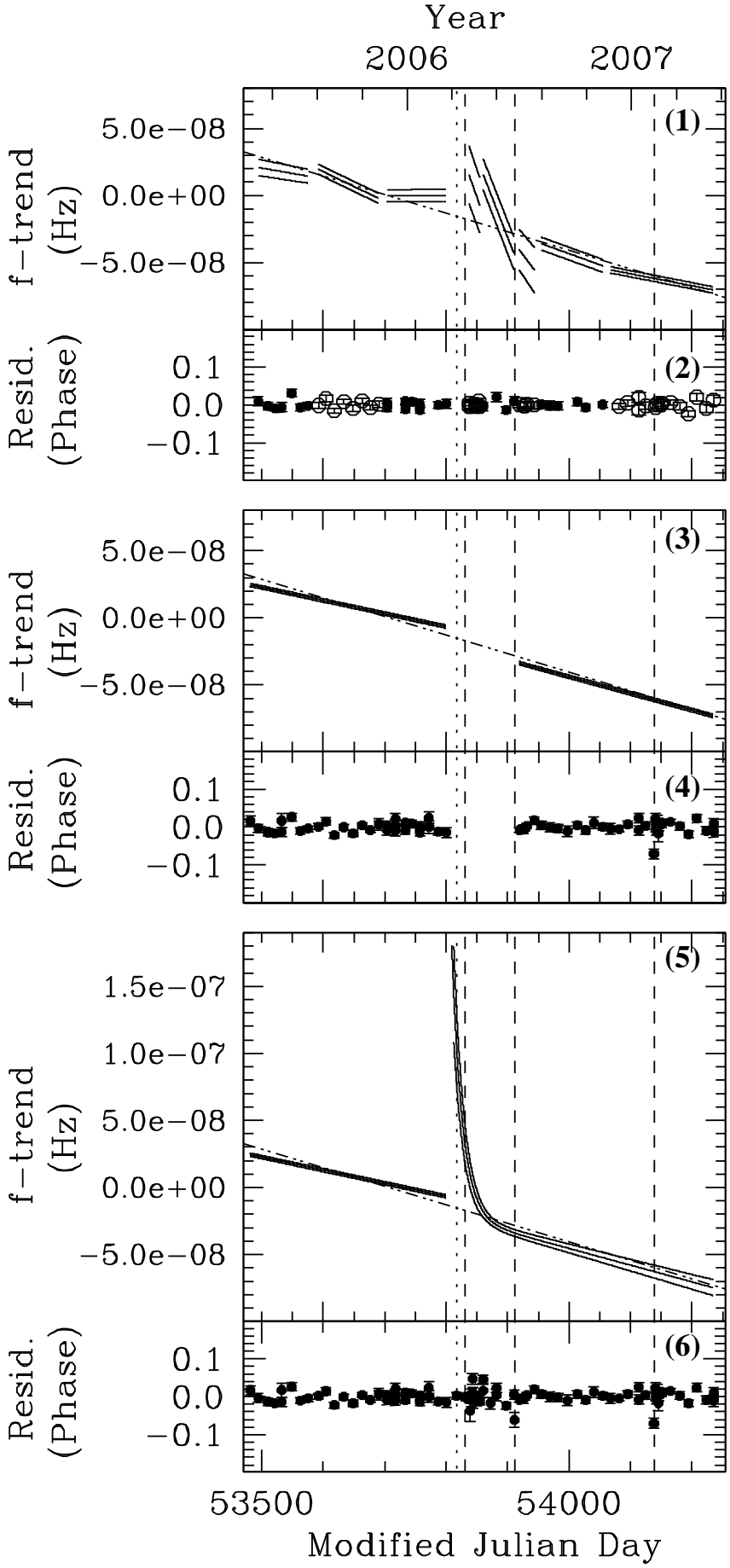

FIG. 12.- Panel 1: Frequency vs. time plot of local linear ephemerides with uncertainties. The linear trend corresponding to the local ephemeris directly before the active phase is subtracted from all local ephemerides. Panel 2: Timing residuals after subtracting the ephemerides shown in panel 1. Panel 3: Frequency vs. time plot (with uncertainties) of the best-fit linear ephemerides in the pre-active phase region and in the post burst 2 region. The same trend as in panel 1 was subtracted. Panel 4: Timing residuals after subtracting the ephemerides shown in panel 3. Panel 5: Frequency vs. time plot (with uncertainties) of the best-fit longterm ephemeris which includes a glitch at MJD 53809 followed by a fast exponential recovery. The same trend as in panel 1 was subtracted. Panel 6: Timing residuals after subtracting the ephemeris shown in panel 5. All panels: the dotted line indicates the epoch of the first observation of the active phase. The dashed lines indicate the burst epochs.
Five of the six bursts reported here for $4 \mathrm{U} 0142+61$ had fast-rise-slow-decay profiles, with tails much longer than the rise times. These morphologies are similar to the class of AXP bursts labeled as "Type B" by Woods et al. (2005). Type B AXP bursts have also been characterized by arriving preferentially at pulse maximum, and are seen more often in AXPs compared with SGRs (Woods et al. 2005; Gavriil et al. 2006). They were suggested as being due to a sudden rearrangement of magnetic field lines anchored in the crust following a crustal fracture (Thompson \& Duncan 1995), as opposed to reconnection events in the upper magnetosphere (e.g. Lyutikov 2002), argued as more likely for the shorter, symmetric "Type A" bursts that show no preference for pulse maximum. However, it is interesting that in the $4 \mathrm{U} 0142+61$ bursts, in spite of their B-type morphology, no clear preference for arrival at or near pulse maximum was seen (see Table 11). This may blur somewhat the distinction between the putative types, although the morphological distinction remains clear.

It is also notable that the vast majority of bursts seen from AXPs had long tails following fast rises. An exception is $1 \mathrm{E} 2259+586$ for which, during its 2002 outburst, the minority (roughly one dozen out of 80) of the bursts seen were of this form, the majority being of Type A, similar to those classically seen in SGRs, and suggested by Woods et al. (2005) to be magnetospheric. A possible hint regarding the origin of the different types may lie in that $1 \mathrm{E} 2259+586$ was mid-outburst when its bursts were observed, whereas the other sources' bursts all occurred in the days/months following the commencement of an active period, presumably as the pulsar recovered from a major event, rather than mid-event.

\subsubsection{Spectral Features}

The complicated spectrum of burst 6 is puzzling. The most broad feature at $\sim 14 \mathrm{keV}$ is particularly interesting. Emission features at similar energies were observed from two out of the three bursts from 1E 1048.1-5937 (Gavriil et al. 2002; Gavriil et al. 2006) and in one out of the four bursts from XTE J1810-197(Woods et al.|2005). We have reanalyzed all these burst spectra consistently as for $4 \mathrm{U} 0142+61$. In Figure 13 we plot the spectra of all AXP bursts with likely emission lines in their spectra. Notice that all have broad features that occur between 13 and $14 \mathrm{keV}$. There is also evidence for a narrow feature at $\sim 8 \mathrm{keV}$ in the $4 \mathrm{U} 0142+61$ burst; there may be hints of additional features in some of the other burst spectra as well.

If the features are interpreted as of a cyclotron origin, it is puzzling that three sources with spin-downinferred magnetic fields that span a range of a factor of three produce features at similar energies. The similarity in energy suggests a mechanism that is not very sensitive to the exact value of the magnetic field. Moreover, it is unclear why such features are seen only in some bursts. Cyclotron features have been observed in the persistent spectra of accretion-powered X-ray pulsars (Coburn et al. 2002). Unlike the features observed here, these features can be well fit by a Gaussian absorption model (see $\S 3.1 .2$ ). In the spectra of some accretionpowered X-ray pulsars there is also evidence for higher harmonics. The features observed in the $4 \mathrm{U} 0142+61$ burst were not consistent with being harmonically re- 


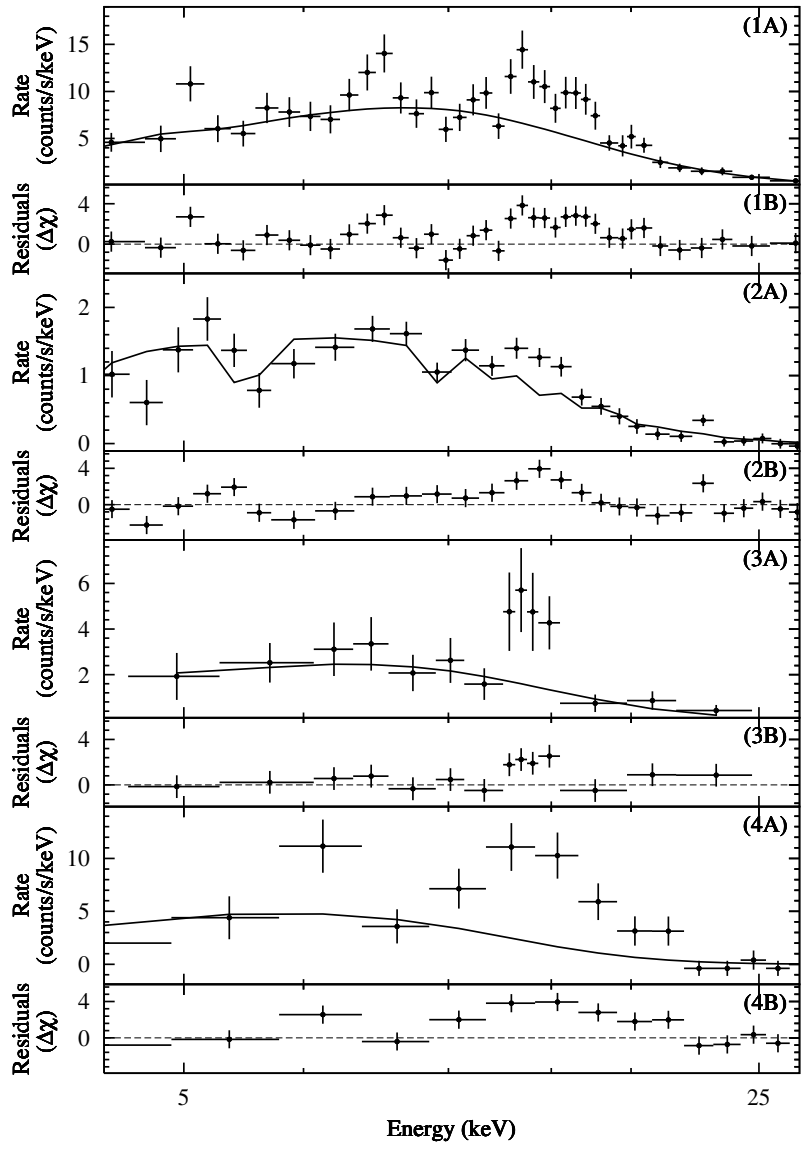

FIG. 13.- Burst spectra of all observed AXP bursts with significant emission lines, as seen by RXTE. Panel 1A: Burst spectrum of $4 \mathrm{U} 0142+61$ burst 6 . The solid line indicates the continuum (blackbody) component of the best-fit model folded through $R X T E$ 's instrumental response. Panel 1B: Residuals, expressed in terms of the change in $\chi^{2}$, after subtracting only the continuum component of the best-fit model. Panels 2A and 2B: Same but for burst 4 of XTE J1810-197 (see Woods et al. 2005). Panels 3A and 3B: Same, but for burst 3 of 1E 1048.1-5937 (see Gavriil et al. 2006). Panels 4A and 4B: Same, but for burst 1 of 1E 1048.1-5937 (see Gavriil et al. 2002).

lated, even after taking into account the relativistic effects on the spacing (see Harding \& Daughtery 1991; Arava \& Harding 1999).

Thus far, all AXP burst spectral features have been observed with $R X T E$. Their absence from many other $R X T E$-observed bursts, together with the absence of any response feature in the PCA near $13-14 \mathrm{keV}$, is supporting evidence for their veracity. Nevertheless, it is important to confirm these features with other instruments. Observations of AXPs during active phases using the Swift XRT or the large-area LAXPC aboard the planned Astrosat mission would be particularly useful in this regard.

\subsection{The Net Spin-Down Event in 4U 0142+61}

The timing glitch reported on in 3.5 had recovery fraction, defined as $Q \equiv \Delta \nu_{d} /\left(\Delta \nu_{d}+\Delta \nu\right)$, equal to $1.07 \pm 0.02$. $Q>1$ implies that the net frequency change after the transients have decayed is negative (see panel 3 of Figure 12 and Table 4 ). Indeed, the net $\Delta \nu / \nu$ observed is $-1.1 \pm 0.1 \times 10^{-7}$. Such a $Q>1$ has not previously been reported in any AXP but has been seen following magnetar-like radiative behavior in one high- $B$ rotation-powered pulsar (Livingstone et al. 2010). Note that Morii et al. (2005) reported a candidate glitch in 1999 from this source. If such a glitch occurred, based on the ephemerides reported in Dib et al. (2007), the fractional frequency change, after any recovery, would have been $+1.9(2) \times 10^{-7}-8.6(2) \times 10^{-7}$ depending on the glitch epoch, but manifestly positive.

In the standard model for glitches in rotation-powered pulsars, the neutron-star crust contains superfluid neutrons that rotate faster than the bulk of the surrounding matter (see, e.g., Anderson \& Itoh 1975; Anderson et al. 1982; Alpar et al. 1984; Alpar \& Pines 1993). This is argued to be a result of the fact that the external magnetic torque acts on the crust and coupled core components only, with the uncoupled superfluid unaffected. The superfluid's angular momentum resides in quantized vortex lines whose density is proportional to angular velocity. The vortex lines are suggested to be pinned to crustal nuclei, and suffer strong forces due to the angular velocity lag that builds between crust and superfluid. A glitch in this picture is a sudden unpinning and outward motion of vortex lines, with a transfer of angular momentum from superfluid to crust. In a magnetar, unpinning may occur due to the strong internal magnetic field as it deforms the crust plastically or cracks it violently (Thompson \& Duncan 1996).

In the $4 \mathrm{U} 0142+61$ event we describe in 93.5 , if the standard glitch model is roughly correct and applies here, then some regions of the stellar superfluid were originally spinning slower than the crust. Then the transient increase in frequency would be a result of transfer of angular momentum first from a more rapidly rotating region, with a subsequent angular momentum drain from the crust to a more slowly rotating region. As argued by Thompson et al. (2000), regions of slowerrotating superfluid can occur in magnetars, because the superfluid vortex motions are governed not be spin-downrelated forces, but by advection across the stellar surface by the deforming crust. Those authors show that the number of vortex lines per unit surface area of crust can increase or decrease depending on the crustal motion relative to the stellar rotation axis. They invoke this possibility to explain a possible "anti-glitch" seen in SGR $1900+14$. However, that event was orders of magnitude larger than what we have observed in $4 \mathrm{U} 0142+61$, with $\Delta \nu / \nu \sim 10^{-4}$ (Thompson et al. 2000), and also was likely associated with the extremely energetic flare seen on 1998 August 27 (Mazets et al. 1999).

Independent evidence for slow crustal deformations in $4 \mathrm{U} 0142+61$ that could result in the regions of slowerrotating superfluid required to explain the spin-down may come from the long-term flux and pulse profile evolution previously reported for this source by Dib et al. (2007) and shown again here in Figures 5 and $6 . \mathrm{Al}-$ though such variations could also be magnetospheric in nature, possibly due to twisting of the magnetic field (e..g. Thompson et al. 2002; Beloborodov \& Thompson 2007), problems with this interpretation exist, as discussed by Dib et al. (2007). For example, if magnetospheric, it is puzzling that most if not all of the changes in pulse profile are seen below $4 \mathrm{keV}$, with none above $6 \mathrm{keV}$. On the other hand, slow evolution of the sur- 
face emission (e.g. Ozzel \& Güver 2007), hence structure, is perhaps more consistent with low-energy evolution. It remains to be seen if detailed modeling can reproduce the sort of slow pulse profile changes we have observed (Fig. 5 ), quite apart from the sudden appearance of new peaks, hence greater harmonic structure, near bursts. We note that the latter has also recently been seen during periods of activity in 1E 1048.1-5937 (Tam et al. 2008).

Previously Kaspi et al. (2000) noted that the existence of AXP glitches having properties comparable to those seen rotation-powered pulsars was not in itself evidence for AXPs being magnetars. This is because the standard glitch model applies regardless of the mechanism by which the crust slows down; i.e., glitches can occur, in principle, in an accretion-powered pulsar. For example, a glitch was a plausible explanation for the fractional change of $\Delta \nu / \nu \sim 4 \times 10^{-5}$ seen in the accreting neutron star system KS 1947+300 (Gallowav et al. 2004). However, although glitches can occur in the case of accreting systems, since there too crust/superfluid angular velocity lags can develop, only in the context of magnetars has the possibility of more slowly rotating superfluid been suggested (Thompson et al. 2000). The net spindown in $4 \mathrm{U} 0142+61$ may thus add to the already large amount of evidence against any accretion-powered origin for $4 \mathrm{U} 0142+61$.

\subsection{The Active Phase of $4 U 0142+61$ and Other AXP Outbursts}

In many ways, the 2006-2007 active phase of $4 \mathrm{U} 0142+61$ is similar to other phases of activity seen in AXPs: it was punctuated by short bursts, pulse profile changes were seen, and it was accompanied by a significant rotational anomaly. However the $4 \mathrm{U} 0142+61$ activity is unique in one interesting way: the pulsar did not suffer a large, long-lived pulse flux increase at the beginning of the phase. Indeed its pulsed flux (Fig. 9 ), apart from very near bursts, has remained relatively stable. We note that observations with focusing X-ray telescopes may reveal some phase-averaged flux variations (see Gonzalez et al. 2010); indeed pulsed fraction has been shown to be inversely correlated with total flux in 1E 1048.1-5937 (Tiengo et al. 2005; Tam et al. 2008), rendering pulsed flux variations smaller relative to total variations. Still, for $4 \mathrm{U} 0142+61$, such a correlation would have to conspire to render the pulsed flux steady. This seems unlikely, however only focusing telescope observations can rule this out.

Other AXP radiative outbursts have been suggested as arising from large magnetospheric twists, with associated magnetospheric currents returning to the stellar surface and heating it, resulting in increased X-ray emission from the source (e.g. Thompson et al. 2002; Beloborodov \& Thompson 2007; Beloborodov 2009). Such twists are thought to represent a release of magnetic energy and helicity from the internally wound-up magnetic field.

On the assumption that the pulsed flux is a reasonable proxy for the total flux for $4 \mathrm{U} 0142+61$, here, we find no clear evidence for significantly increased X-ray emission on time scales longer than a few minutes. Thus any largescale magnetospheric twist scenario is problematic. As originally showed by Thompson et al. (2002), the X-ray luminosity due to returning currents reheating the sur- face in a significantly twisted magnetosphere in general are comparable to that produced from internal processes; this is clearly not observed in the 2006-7 active period of $4 \mathrm{U} 0142+61$.

Instead, for this source, long-term evolution of the crust, driven presumably internally by field decay, and resulting in multiple unstable configurations though without any large scale magnetospheric twists, could result in sudden cracking and local rearrangements. This could be accompanied by bursts and profile changes, as well as with vortex line shifting. Why such surface motion does not produce significant magnetospheric twists is, however, puzzling, given that field lines are thought to be anchored in the crust; perhaps only for large motions do field lines become sufficiently twisted for enhanced X-ray emission to be produced.

Dib et al. (2008) showed that other AXP glitches have been unaccompanied by radiative changes. For example, AXP 1E 1841-045 has glitched multiple times yet its pulsed flux remains very steady. Such radiatively "silent" glitches may be a result of internal activity that does not result in significant twisting of outer magnetosphere field lines, where as radiatively "loud" glitches could be those for which the magnetosphere is impacted. We note in fact that thus far, the data are consistent with all AXP radiative outbursts being accompanied by timing anomalies. This may also be true of SGRs, though in those cases, timing anomalies are harder to establish because of the difficulty in achieving phase-coherent timing in quiescence.

\section{SUMMARY AND CONCLUSIONS}

We have reported on anomalous X-ray pulsar $4 \mathrm{U} 0142+61$ entering an active phase which was preceded with by a long-term increase in pulsed flux. The active phase, which commenced in 2006 March, consisted of a timing anomaly that can be described as a net "antiglitch," that is, a net spin-down following an initial spinup that decayed on a time scale of 17 days. Following the glitch, we detected six bursts from the pulsar, the first ever observed from this source. Despite $10 \mathrm{yr}$ of RXTE monitoring, the bursts all occurred in the narrow time span between 2006 April 6 and 2007 February 7. The sixth and largest burst had a unusual spectrum that cannot be described by any simple continuum model. Rather, it can be well fit if a broad spectral feature at $\sim 14 \mathrm{keV}$ is included, as has been reported in other AXP bursts, as well as a narrow feature at $\sim 8 \mathrm{keV}$. The pulse profile of the source changed from double- to triple- peaked near the bursts, and underwent considerable evolution otherwise. At the burst epochs the relative intensity of the three peaks significantly varied. The pulse profile is now relaxing to its pre-active phase morphology. Most aspects of 4U 0142+61's emission changed during the active phase, with the notable exception of the pulsed flux (except near bursts). This argues against this event being associated with a sudden magnetospheric twist, as has been invoked for other AXP activity, and is suggestive of crustal evolution driven internally by the large magnetic field, though without significant magnetospheric twisting. We suggest that other, radiatively silent AXP glitches have a similar origin, whereas radiatively loud AXP timing events occur when the crustal motions cannot avoid significant twisting of the magnetic 
field lines.

We thank P. M. Woods and A. M. Beloborodov for useful discussions. This research has made use of data obtained through the High Energy Astrophysics Science Archive Research Center Online Service, provided by the NASA/Goddard Space Flight Center. This work has been supported by an NSERC Discovery Grant, the Canadian Institute for Advanced Research, and Le Fonds Québécois de la Recherche sur la Nature et les Technologies, by the Canada Research Chairs program, and by the Lorne Trottier Chair in Observational Astrophysics.

\section{REFERENCES}

Alpar, M. A., Anderson, P. W., Pines, D., \& Shaham, J. 1984, ApJ, 276, 325

Alpar, M. A. \& Pines, D. 1993, in Isolated Pulsars, ed. R. E. K. A. van Riper \& C. Ho (Cambridge University Press), 17-27

Anderson, P. W., Alpar, M. A., Pines, D., \& Shaham, J. 1982, Phil Mag A, 45, 227

Anderson, P. W. \& Itoh, N. 1975, Nature, 256, 25

Araya, R. A., \& Harding, A. K. 1999, ApJ, 517, 334

Beloborodov, A. M. 2009, ApJ, 703, 1044

Beloborodov, A. M. \& Thompson, C. 2007, Ap\&SS, 308, 631

Coburn, W., Heindl, W. A., Rothschild, R. E., Gruber, D. E., Kreykenbohm, I., Wilms, J.,Kretschmar, P., \& Staubert, R. 2002, ApJ, 580, 394

Dib, R., Kaspi, V. M., \& Gavriil, F. P. 2007, ApJ, 666, 1152

-. 2008, ApJ, 673, 1044

—. 2009, ApJ, 702, 614

Durant, M. \& van Kerkwijk, M. H. 2006, ApJ, 650, 1070

Durant, M. \& van Kerkwijk, M. H. 2006, ApJ, 650, 1082

Galloway, D. K., Morgan, E. H., \& Levine, A. M. 2004, ApJ, 613, 1164

Gavriil, F. P. \& Kaspi, V. M. 2002, ApJ, 567, 1067

-. 2004, ApJ, 609, L67

Gavriil, F. P., Kaspi, V. M., \& Woods, P. M. 2002, Nature, 419, 142

-. 2004, ApJ, 607, 959

Gavriil, F. P., Kaspi, V. M., \& Woods, P. M. 2006, ApJ, 641, 418

Gonzalez, M. E., Dib, R., Kaspi, V. M., Woods, P. M., Tam, C. R., \& Gavriil, F. P. 2010, ApJ, 716, 1345

Gotthelf, E. V. \& Vasisht, G. 1998, New Astronomy, 3, 293

Halpern, J. P., Gotthelf, E. V., Reynolds, J., Ransom, S. M., \& Camilo, F. 2008, ApJ, 676, 1178

Harding, A. K., \& Daugherty, J. K. 1991, ApJ, 374, 687

Ibrahim, A. I., Markwardt, C. B., Swank, J. H., Ransom, S., Roberts, M., Kaspi, V., Woods, P. M., Safi-Harb, S., Balman, S., Parke, W. C., Kouveliotou, C., Hurley, K., \& Cline, T. 2004, ApJ, 609, L21

Israel, G. L., Campana, S., Dall'Osso, S., Muno, M. P., Cummings, J., Perna, R., \& Stella, L. 2007a, ApJ, 664, 448

Israel, G. L., Götz, D., Zane, S., Dall'Osso, S., Rea, N., \& Stella, L. 2007b, A\&A, 476, L9

Jahoda, K., Markwardt, C. B., Radeva, Y., Rots, A. H., Stark, M. J., Swank, J. H., Strohmayer, T. E., \& Zhang, W. 2006, ApJS, 163, 401

Kaspi, V. M. 2007, Ap\&SS, 308, 1

Kaspi, V. M., Gavriil, F. P., Woods, P. M., Jensen, J. B., Roberts, M. S. E., \& Chakrabarty, D. 2003, ApJ, 588, L93

Kaspi, V. M., Lackey, J. R., \& Chakrabarty, D. 2000, ApJ, 537, L31
Krimm, H., Barthelmy, S., Campana, S., Cummings, J., Israel, G., Palmer, D., \& Parsons, A. 2006, The Astronomer's Telegram, 894, 1

Kumar, H. S. \& Safi-Harb, S. 2011, ApJ, 725, L191

Livingstone, M. A., Kaspi, V. M., \& Gavriil, F. P. 2010, ApJ, 710,1710

Lyutikov, M. 2002, ApJ, 580, L65

Mazets, E. P., Cline, T. L., Aptekar', R. L., Butterworth, P. S., Frederiks, D. D., Golenetskii, S. V., Il'Inskii, V. N., \& Pal'Shin, V. D. 1999, Astronomy Letters, 25, 635

Mereghetti, S. 2008, A\&A Rev., 15, 225

Mereghetti, S., Götz, D., Weidenspointner, G., von Kienlin, A., Esposito, P., Tiengo, A., Vianello, G., Israel, G. L., Stella, L. Turolla, R., Rea, N., \& Zane, S. 2009, ApJ, 696, L74

Mihara, T. 1995, Ph.D. thesis, Univ. Tokyo

Morii, M., Kawai, N., \& Shibazaki, N. 2005, ApJ, 622, 544

Muno, M. P., Gaensler, B. M., Clark, J. S., de Gr ijs, R., Pooley, D., Stevens, I. R., \& Portegies Zwart, S. F. 2007, MNRAS, 378, L44

Özel, F. \& Güver, T. 2007, ApJ, 659, L141

Patel, S. K., Kouveliotou, C., Woods, P. M., Tennant, A. F., Weisskopf, M. C., Finger, M. H., Wilson, C. A., Göğüs, E., van der Klis, M., \& Belloni, T. 2003, ApJ, 587, 367

Tam, C. R., Gavriil, F. P., Dib, R., Kaspi, V. M., Woods, P. M., \& Bassa, C. 2008, ApJ, 677, 503

Tam, C. R., Kaspi, V. M., Gaensler, B. M., \& Gotthelf, E. V. 2006, ApJ, 652, 548

Thompson, C. \& Duncan, R. C. 1995, MNRAS, 275, 255

Thompson, C. \& Duncan, R. C. 1996, ApJ, 473, 322

Thompson, C., Duncan, R. C., Woods, P. M., Kouveliotou, C., Finger, M. H., \& van Paradijs, J. 2000, ApJ, 543, 340

Thompson, C., Lyutikov, M., \& Kulkarni, S. R. 2002, ApJ, 574, 332

Tiengo, A., Mereghetti, S., Turolla, R., Zane, S., Rea, N., Stella, L., \& Israel, G. L. 2005, A\&A, 437, 997

White, N. E., Swank, J. H., \& Holt, S. S. 1983, ApJ, 270, 711

Woods, P. M., Kaspi, V. M., Thompson, C., Gavriil, F. P.

Marshall, H. L., Chakrabarty, D., Flanagan, K., Heyl, J., \& Hernquist, L. 2004, ApJ, 605, 378

Woods, P. M., Kouveliotou, C., Göğüs, E., Finger, M. H., Swank, J., Smith, D. A., Hurley, K., \& Thompson, C. 2001, ApJ, 552, 748

Woods, P. M., Kouveliotou, C., Gavriil, F. P., Kaspi, M., V., Roberts, M. S. E., Ibrahim, A., Markwardt, C. B., Swank, J. H., \& Finger, M. H. 2005, ApJ, 629, 985

Woods, P. M. \& Thompson, C. 2006, in Compact Stellar X-ray Sources, ed. W. H. G. Lewin \& M. van der Klis (UK: Cambridge University Press)

Zhu, W., Kaspi, V. M., Dib, R., Woods, P. M., Gavriil, F. P., \& Archibald, A. M. 2008, ApJ, 686, 520 